\title{
Factores incidentes en el rendimiento académico estudiantil de Ingeniería en Sistemas de Información de la FAREM-Matagalpa, 2012-2016
}

\author{
Guiselle Raquel Martínez Ramos ${ }^{1}$ \\ Manuel Enrique Pedroza Pacheco²
}

\section{RESUMEN}

El propósito de este estudio es describir factores que inciden en el rendimiento académico de estudiantes de Ing. Sistemas de Información de la FAREM Matagalpa, clasificados como personales, sociales e institucionales. El estudio es mixto, exploratorio y descriptivo, observacional, retrospectivo y transversal. La muestra de estudio fue por conveniencia. Las técnicas de recolección fueron análisis documental y protocolo de encuesta. Se procesaron datos estadísticamente con modelos ANACORR, ANARE, ACP, tablas de contingencia y frecuencia, gráficos de barra, boxplot y pastel para describir resultados. Se concluye que la mayoría de estudiantes desertaron de la carrera en primer año, los estudiantes activos mejoraron levemente el rendimiento cada año, factores personales relevantes fueron competencia cognitiva, motivación, atribuciones causales, bienestar psicológico, asistencia a clases e inteligencia emocional, no existe relación entre promedio de secundaria y de universidad, la última no se asocia con la nota de admisión, factores sociales relevantes fueron entorno familiar, nivel educativo de padres, capital cultural y contexto socioeconómico; factores institucionales determinantes fueron complejidad de estudios, condiciones institucionales, servicios de apoyo, relación estudiante-docente y pruebas de admisión. Se recomienda tomar resultados de esta investigación para profundizar en el tema y mejorar el rendimiento académico de los estudiantes universitarios.

Palabras clave: rendimiento académico, personales, sociales, institucionales.

Recibido: 30 de marzo de 2017

Aceptado: 21 de septiembre de 2017

1 Docente UNAN-Managua. FAREM-Matagalpa. Estudiante de doctorado en Gestión y Calidad de la Investigación Científica. UNAN-Managua, FAREM-Estelí. Correo Electrónico: mat guisselle@hotmail.com

2 Profesor titular UNAN-Managua. Coordinador de Doctorado en Gestión y Calidad de la Investigación. UNAN-Managua. FAREM-Estelí. Correo electrónico: hppedroza@gmail.com 


\title{
Factors that affect students academic performance in Information Systems Engineering, FAREM-Matagalpa, 2012-2016
}

\begin{abstract}
The purpose of this study is to describe factors that affect the academic performance of students of Ing. Information Systems of FAREM Matagalpa, classified as personal, social and institutional. The study is mixed, exploratory and descriptive, observational, retrospective and transversal. The study sample was for convenience. The collection techniques were documentary analysis and survey protocol. Statistical data were processed with ANACORR, ANARE, ACP, contingency and frequency tables, bar graphs, boxplot and pie chart to describe results. It is concluded that the majority of students dropped out of the first year, active students slightly improved performance each year, relevant personal factors were cognitive competence, motivation, causal attributions, psychological well-being, class attendance and emotional intelligence, there is no relationship between secondary and university average, the last one is not associated with the admission grade, relevant social factors were family environment, educational level of parents, cultural capital and socioeconomic context; Institutional determinants were complexity of studies, institutional conditions, support services, student-teacher relationship and admission tests. It is recommended to take results of this research to deepen the subject and improve the academic performance of university students.
\end{abstract}

Keywords: academic performance, personal, social, institutional. 


\section{INTRODUCCIÓN}

En la actualidad, el sistema educativo mide la calidad estudiantil con las calificaciones obtenidas durante el desarrollo de los cursos estudiados, que a la vez, son la base para decidir si un estudiante aprueba o no los mismos; este conjunto de calificaciones definen si el rendimiento académico del grupo y del estudiante mismo es aceptable o no para aprobar un curso. Así, el rendimiento académico es un factor muy importante en el quehacer educativo, ya que el mismo rige en gran proporción el éxito o fracaso escolar, y es dado a conocer por medio de informes presentados en cada corte evaluativo.

A nivel universitario, es preocupante el porcentaje de estudiantes reprobados, que propicia un índice de deserción masivo en las universidades, por ello, el rendimiento académico es un tema de mucho interés investigativo, ya que un bajo rendimiento afecta varios agentes; la familia del estudiante y el gobierno tendrán que invertir más dinero en semestres académicos adicionales y el estudiante deberá esperar más tiempo para graduarse e insertarse en la sociedad laboral.

En muchas instituciones educativas de Nicaragua, el rendimiento académico ha sido sinónimo de la calidad educativa de los centros como tal, sin embargo, es de vital importancia conocer los factores determinantes de tal fenómeno, ya que es el punto de partida para actuar en pro de la calidad del aprendizaje y por ende para un mejor rendimiento académico, dichos factores han sido estudiados en todo el mundo, autores como (Gómez, Oviedo, \& Martínez, 2011, pág. 91) afirman que "el rendimiento académico no es el producto de una única capacidad, sino el resultado sintético de una serie de factores que actúan en, y desde, la persona que aprende".

En la Universidad Nacional Autónoma de Nicaragua (UNAN Managua), se realizó un análisis del rendimiento académico en todas las carreras que se sirven en cada facultad de esta alma máter; donde un
$62.35 \%$ aprobó todas las asignaturas y el porcentaje restante reprobó al menos una. En todas las facultades, a través de las direcciones de departamentos docentes se orientó realizar un análisis reflexivo sobre los resultados obtenidos, además de sugerencias de mejora desde los colectivos docentes. Aunque los resultados muestran más del sesenta por ciento de aprobados, no deja de preocupar el porcentaje restante de estudiantes reprobados (Universidad Nacional Autónoma de Nicaragua, 2016).

En la Facultad Regional Multidisciplinaria de Matagalpa(FAREM Matagalpa), de la UNAN Managua se obtuvo un $65.09 \%$ de aprobados y un $34.91 \%$ de estudiantes que reprobaron al menos una asignatura. Carreras como Ing. en Sistemas de Información e Ing. en Ciencias de la Computación muestran un índice mayor a la media de reprobados, la primera presentó un $44 \%$ de estudiantes reprobados y la segunda un $52 \%$ (Ver anexo 2). Los resultados presentados son apenas un indicador resultante de múltiples factores que inciden de manera directa o indirecta en el rendimiento académico de los estudiantes, lo que indica la importancia que tiene indagar a profundidad los factores que influyen directa e indirectamente en el comportamiento de los estudiantes con respecto a su quehacer académico (Universidad Nacional Autónoma de Nicaragua, 2016).

Cabe mencionar que la carrera Ing. en Sistemas de Información fue ofertada por primera vez en la FAREM Matagalpa en el año 2010 y se dejó de ofertar en el 2013 para ofertar la carrera Ing. Ciencias de la Computación, la que fue cerrada en el 2015. En el 2016 se ofertó nuevamente la primera carrera mencionada, lo que da mayor importancia a conocer el comportamiento de rendimiento académico que se obtuvo en el periodo 2010 - 2016, con el propósito de identificar los factores que incidieron en la calidad del rendimiento académico de los estudiantes de Ing. en Sistemas de Información en el periodo mencionado. 
Estudios similares se han realizado a nivel mundial en todos los niveles de educación, donde se identifican factores predominantes que afectan el desempeño de los estudiantes en el ámbito académico. Autores como (Cuasapaz \& Rubio, 2013) realizaron un estudio con el propósito de mejorar el rendimiento académico de los niños de la escuela de educación básica Sucre, clasificando los factores como familiares, pedagógicos, psicológicos, fisiológicos, económicos y sociales. (Torres \& Rodríguez, 2006), realizaron un estudio con estudiantes universitarios para examinar sus contextos universitario y familiar; abordaron información relacionada con factores escolares, familiares, personales, sociales y culturales.

(Tejedor \& García, 2007), presentan las opiniones de profesores y estudiantes sobre las causas del bajo rendimiento universitario de forma comparativa, clasifican dichas causas en las categorías institucionales, relacionadas con el profesor y el estudiante. (Gómez, Oviedo, \& Martínez, 2011), verificaron si existe relación del rendimiento académico con las variables sexo, carrera y semestre, además del promedio y la satisfacción con la carrera elegida. (Garbanzo, 2007), señala los posibles factores asociados al rendimiento académico en estudiantes universitarios, y su vinculación con la calidad de la educación superior pública en general,categorizándolos en determinantes personales, sociales e institucionales, clasificación que otros autores como (Miñano, Cantero, \& Castejón, 2008) han adoptado, dichos autores se han basado en lo expuesto por (Castejón \& Pérez, 1998).

Todos los estudios citados anteriormente, tienen un objetivo en común con esta investigación, ya que se indaga sobre los factores que inciden en el rendimiento de los estudiantes, clasificados de diversas formas pero encaminado a caracterizar los factores más determinantes en el rendimiento académico. Las clasificaciones realizadas por (Garbanzo, 2007); (Miñano, Cantero, \& Castejón, 2008) y (Castejón \& Pérez, 1998), es de interés para este estudio, ya que aporta información más detallada sobre el tema y una clasificación más acorde con el entorno del estudiante, desde las perspectivas social, institucional y personal, esto ayudará a definir mejor qué factores tienen más incidencia en el rendimiento académico de los estudiantes, lo que orientará de una manera más acertada cuáles se deben analizar para realizar el estudio pertinente en pro de mejorar la situación estudiada.

\section{MATERIALES Y MÉTODOS}

\section{Tipo de Estudio}

Por el tipo de estudio, esta investigación es descriptiva, ya que se basó en el método de la observación del comportamiento del rendimiento académico y la descripción de los factores identificados (Piura, 2006). Por su enfoque filosófico es un estudio mixto, porque se establecen relaciones entre variables cuantitativas y cualitativas y se valoraron opiniones diversas de los estudiantes de la muestra, según el método utilizado es observacional, porque se describieron los resultados tal y como se dieron (Hernández, Fernández, \& Baptista, 2010). Según el corte en el tiempo, es transversal, debido al periodo y secuencia del estudio, comprendido en los meses de noviembre y diciembre 2016, y es retrospectivo porque se estudiaron los hechos ocurridos en años anteriores contemplados entre 2010 y 2016 (Pineda, de Alvarado, \& de Canales, 1994).

Área de estudio

El estudio se realizó en la carrera de Ing. en Sistemas de información, en la Facultad Regional Multidisciplinaria de Matagalpa (FAREM, Matagalpa), fue dirigida a estudiantes activos de la carrera mencionada, pertenecientes a la cohorte $2012-2016$

\section{Universo y Muestra}

Según los datos facilitados por la Secretaría de Facultad de la FAREM Matagalpa, la cantidad de estudiantes que se han matriculado en la carrera de Ing. en Sistemas es 100. Para el estudio se tomó una muestra no probabilística decisional, seleccionando como sujetos de investigación a 28 estudiantes activos en la carrera. 
Métodos, técnicas e instrumentos de recolección de datos

Se realizó un análisis documental con la información concerniente a las calificaciones obtenidas, asignaturas matriculadas y asignaturas reprobadas por cada estudiante, clasificadas por año académico, abarcando el periodo 2010-2016. Esta información permitió contabilizar la promoción, deserción y retención de los estudiantes de la carrera de ingeniería en sistemas de información. Se diseñó una encuesta para identificar los factores que inciden en el rendimiento académico y clasificada en determinantes personales, sociales e institucionales. Dicha encuesta fue dirigida a los estudiantes activos de la cohorte 2012-2016 de la carrera mencionada. Este instrumento permitió identificar los factores que inciden en el rendimiento académico de los encuestados.

\section{Procedimientos para la recolección de datos e información}

Para la aplicación de la encuesta se recopiló el correo electrónico de cada estudiante de la muestra y se utilizó la aplicación en Google Forms para que se respondiera dicha encuesta en línea, se contactó a algunos estudiantes en el aula de clase y a los demás telefónicamente, se les solicitó respondieran la encuesta a la brevedad.

\section{Métodos, técnicas e instrumentos de recolección de datos}

Se realizó un análisis documental con la información concerniente a las calificaciones obtenidas, asignaturas matriculadas y asignaturas reprobadas por cada estudiante, clasificadas por año académico, abarcando el periodo 2010-2016. Esta información permitió contabilizar la promoción, deserción y retención de los estudiantes de la carrera de ingeniería en sistemas de información.Se diseñó una encuesta para identificar los factores que inciden en el rendimiento académico y clasificada en determinantes personales, sociales e institucionales. Dicha encuesta fue dirigida a una muestra por conveniencia que abarca la cantidad de estudiantes activos de la carrera mencionada, con un total de 28. Este instrumento permitió identificar los factores que inciden en el rendimiento académico de los encuestados (Hernández, Fernández, \& Baptista, 2010).

\section{Procedimientos para la recolección de Datos e Información}

Para la aplicación de la encuesta se recopiló el correo electrónico de cada estudiante de la muestra y se utilizó la aplicación en Google Forms para que se respondiera dicha encuesta en línea, se contactó a algunos estudiantes en el aula de clase y a los demás telefónicamente, se les solicitó respondieran la encuesta a la brevedad.

\section{Tabulación y análisis estadísticos de los Datos}

Para la tabulación de la información, se exportaron los datos a una hoja de excel, se codificaron y posteriormente se procesaron en los softwares estadísticos Infostat versión 2016p e IBM SPSS Statistics v. 20.

Se analizaron descriptivamente las variables nominales y numéricas; se realizaron análisis de frecuencia y se mostraron las tablas correspondiente, se diseñaron gráficos depastel, barras para variables de categorías, gráficos de barra para variables dicotómicas para describir resultados de múltiples factores en un mismo plano cartesiano, se utilizó el gráfico de cajas y bigotes para describir resultados variables numéricas continuas y discretas.

Se realizaron los Análisis de Contingencia pertinentespara algunas variables no paramétricas, se aplicó la prueba de Correlación no Paramétrica de Tau C de Kendall para demostrar la correlación lineal entre variables de categorías, además de la prueba de correlación de Pearson y la Regresión Lineal para variables numéricas continuas; todos los resultados se compararon con el nivel de significancia prestablecido para la prueba entre ambos factores, de manera que cuando $\mathrm{p} \leq 0.05$ no se aceptará la hipótesis nula planteada de $\rho=0$. Para todos los análisis descritos se utilizó SPSS v. 20. 
Para las variables pertinentes a los determinantes personales, sociales e institucionales,se aplicó la técnica de Análisis Multivariados Análisis de Componente Principal (ACP), cuyo criterio de clasificación utilizado fue el sexo de los estudiantes y las variables combinadas fueron los determinantes clasificados por separado. Los análisis inferenciales antes descritos, se obtuvieron utilizando el software estadístico Infostat $\mathrm{v}$ 2016p para Windows, de acuerdo a los procedimientos estadísticos descritos en (Pedroza \& Dicovskyi, 2006).

\section{RESULTADOS Y DISCUSIÓN}

En este apartado se abordarán los resultados obtenidos a partir del análisis documental y la encuesta aplicada para describir el comportamiento del rendimiento académico de los estudiantes de la carrera Ing. en Sistemas de Información de la FAREM Matagalpa, de la UNAN Managua e identificar los factores que inciden en mismo.

Se realizaron los Análisis de Contingencia pertinentes paraalgunas variablesnoparamétricas, seaplicólaprueba de Correlación no Paramétrica de Tau C de Kendall para demostrar la correlación lineal entre variables de categorías, además de la prueba de correlación de Pearson y la Regresión Lineal para variables numéricas continuas; todos los resultados se compararon con el nivel de significancia prestablecido para la prueba entre ambos factores, de manera que cuando $\mathrm{p} \leq 0.05$ no se aceptará la hipótesis nula planteada de $\rho=0$. Para todos los análisis descritos se utilizó SPSS v. 20.

Para las variables pertinentes a los determinantes personales, sociales e institucionales, se aplicó la técnica de Análisis Multivariados Análisis de Componente Principal (ACP), cuyo criterio de clasificación utilizado fue el sexo de los estudiantes y las variables combinadas fueron los determinantes clasificados por separado. Los análisis inferenciales antes descritos, se obtuvieron utilizando el software estadístico Infostat v 2016p para Windows, de acuerdo con los procedimientos estadísticos descritos en (Pedroza (a), 2014)

\section{Caracterización del Grupo de Ing. en Sistemas de Información}

Los estudiantes de la carrera de Ing. en Sistemas de Información 2010-2016, tienen edades entre los 21 y 25 años, la mitad de ellos están entre los 23 y 24 años de edad (Figura 1); de acuerdo al sexo son mujeres en su mayoría (Figura 2), casi todos son solteros (Figura 3), la mayor parte proceden de centros educativos públicos (Figura 4), menos de la mitad residen en la zona urbana y la minoría en la zona rural (Figura 5), la gran mayoría no trabaja (Figura 6) y aún viven con sus padres (Figura 7).

Los resultados predicen que la mayor parte de los estudiantes poseen características adecuadas para cursar sin problemas cualquier carrera que quisieran estudiar, son jóvenes, no tienen responsabilidades maritales ni laborales, residentes en zonas con todos los servicios básicos y son subsidiados por sus padres.

Los resultados predicen que la mayor parte de los estudiantes poseen características adecuadas para cursar sin problemas cualquier carrera que quisieran estudiar, son jóvenes, no tienen responsabilidades maritales ni laborales, residentes en zonas con todos los servicios básicos y son subsidiados por sus padres.

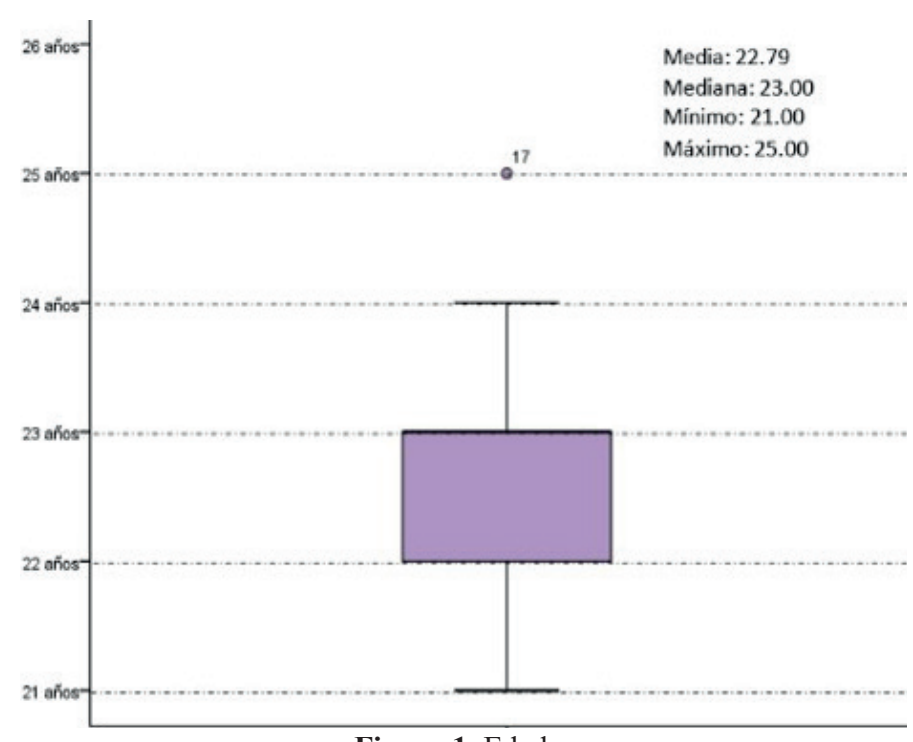

Figura 1. Edades 


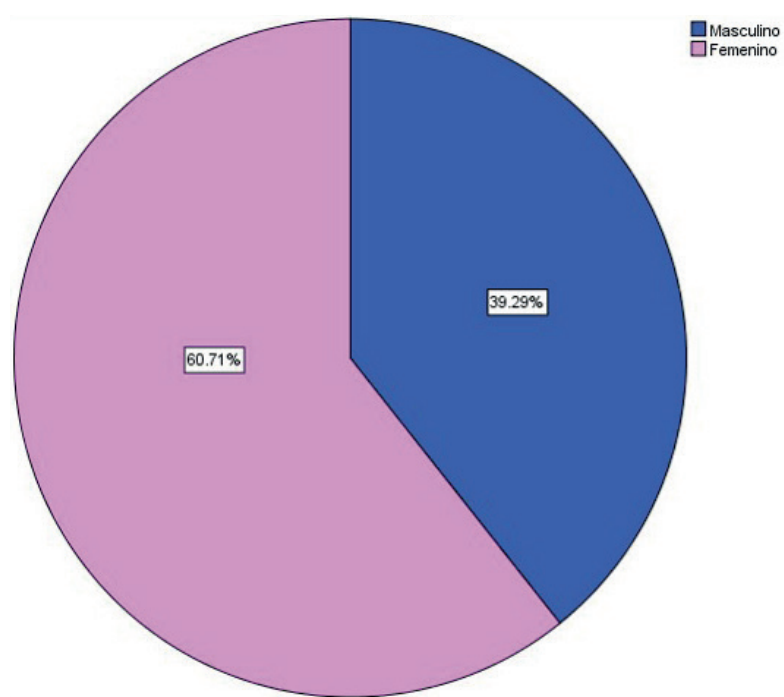

Figura 2. Sexo

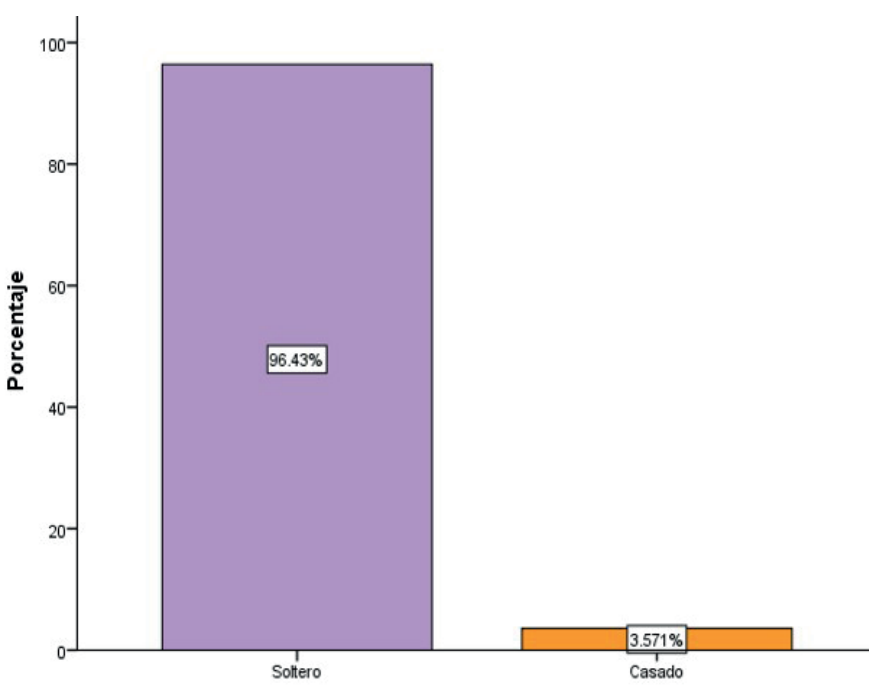

Figura 3. Estado Civil

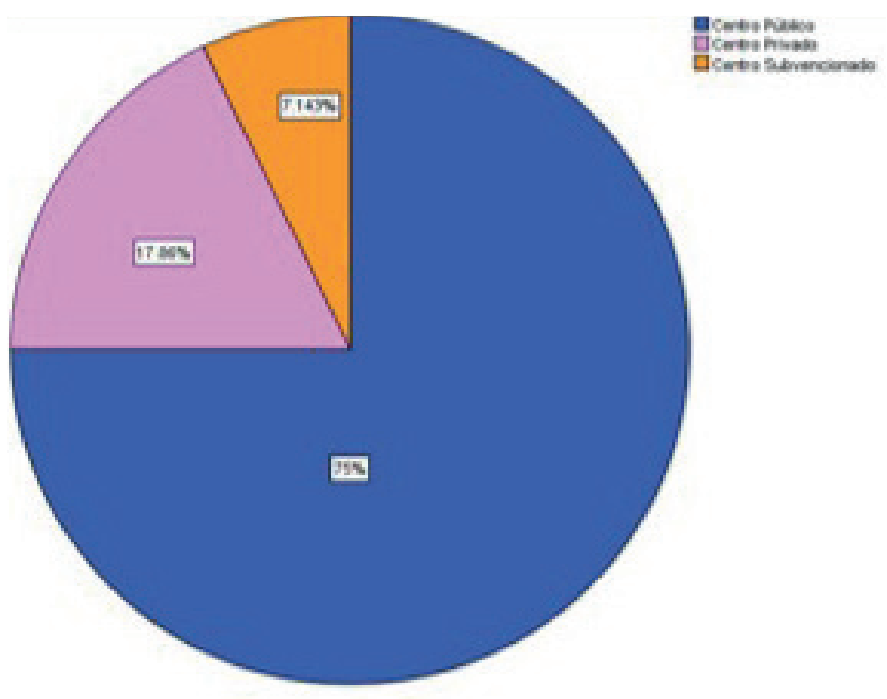

Figura 4. Tipo de Centro de Procedencia

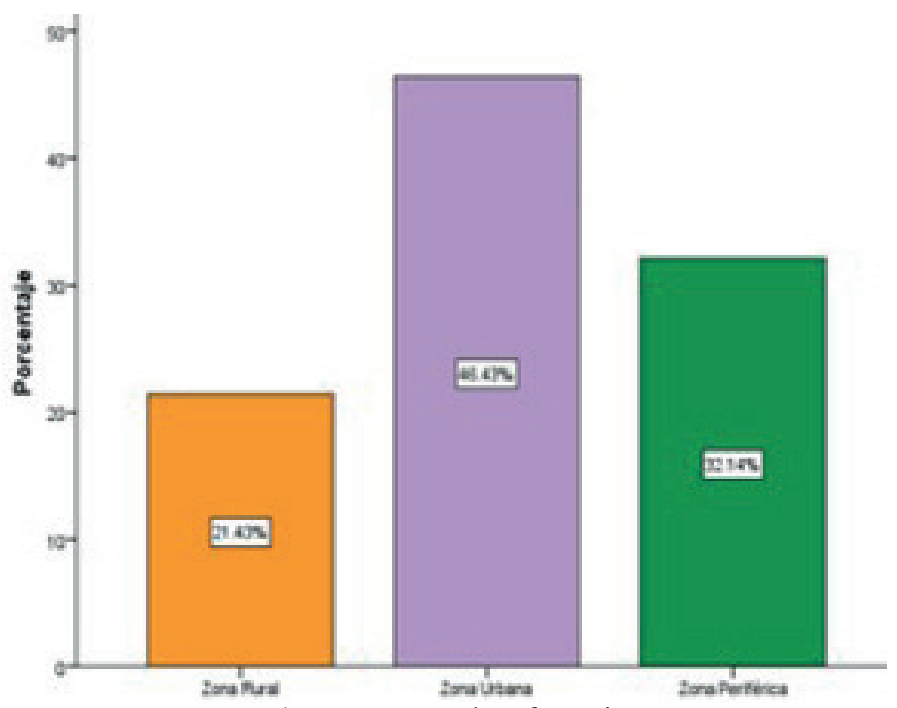

Figura 5. Zona de referencia

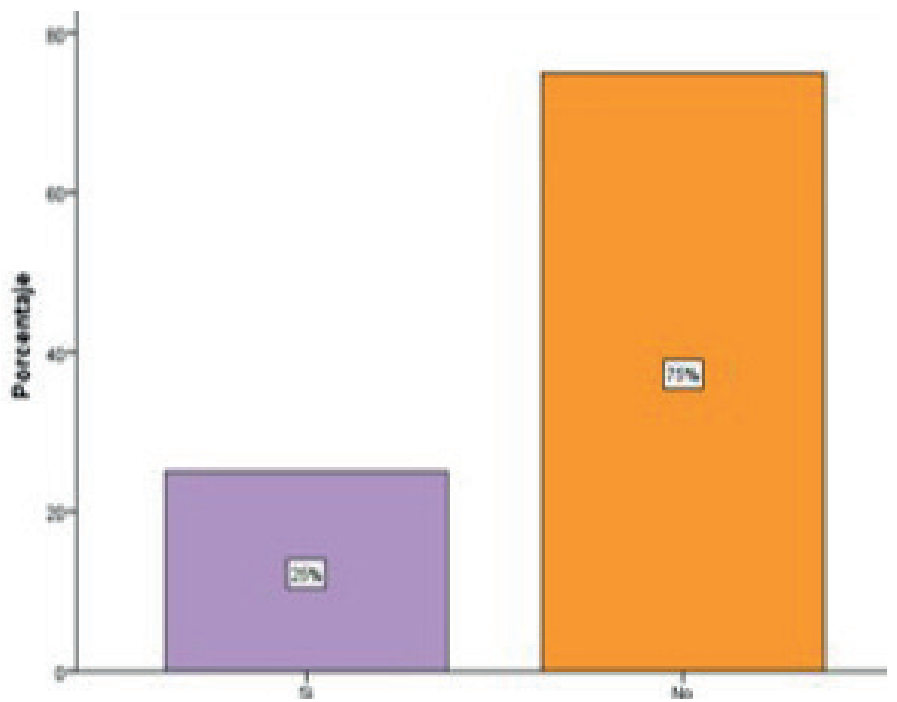

Figura 6. Condición Laboral

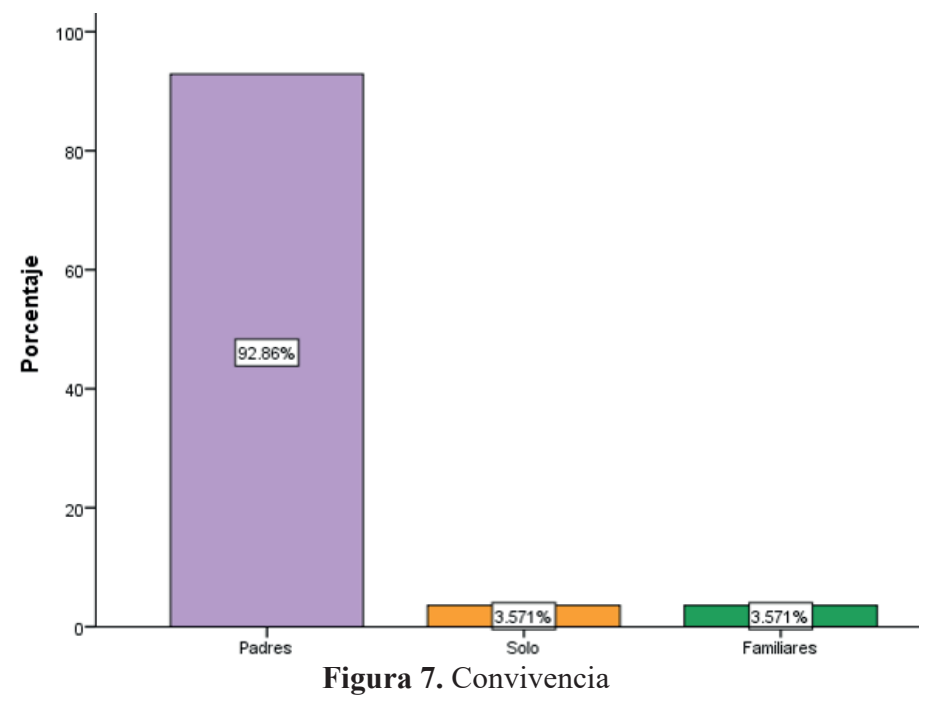

Fuente: Propia a partir de resultados de encuesta aplicada a estudiantes $y$ datos proporcionados por secretaría de facultad 


\section{Comportamiento del Rendimiento Académico}

El análisis documental reveló que en los años 2010, 2011 y 2012 se matricularon 100 estudiantes en la carrera de Ing. Sistemas de Información, el cupo para esta carrera era de 40 debido a la capacidad de los laboratorios, cabe mencionar que en la actualidad la cantidad de estudiantes que se matriculan es de 50 . Algunos estudiantes clasificaron en esta carrera, se matricularon, pero no cursaron ninguna asignatura, esta puede ser la razón por la que aparecen 100 estudiantes en los registros del sistema de registro académico.

Según informes del Sistema de Registro Académico, suministrados por la Secretaría de Facultad, de los 100 estudiantes matriculados en los periodos 2010-2016, (Cuadro 1), han egresado 25 estudiantes, esto es, según (Vado, Mojica, \& Gómez, 2014), que especifican que es la cantidad de estudiantes que han cumplimentado los requisitos de acreditación correspondientes al plan de estudios establecido para culminar la carrera.

28 estudiantes aún están activos, esta cantidad más los egresados conforman el porcentaje de retención, que equivale a un $53 \%$ de los estudiantes inicialmente matriculados; para (Guido, 2016), la retención es la capacidad del sistema educativo para lograr la permanencia de los alumnos en las aulas, garantizando la culminación de niveles en los tiempos correspondientes, asegurando el dominio de las competencias y conocimientos correspondientes. La retención en esta carrera es apenas un poco más de la mitad de los estudiantes matriculados, lo que hace sospechar que hay factores relevantes que están provocando que los estudiantes abandonen esta carrera y que podrían ser objeto de un estudio interesante que podría ayudar a disminuir la deserción universitaria.

Lo anterior significa que un $47 \%$ de los estudiantesde desertó de la carrera en diferentes años académicos, 24 de ellos desertaron en el primer año, 8 en el segundo año, 9 en tercero, 2 en cuarto y 3 en quinto año; lo que indica que en el primer año se da un fenómeno determinante para que los universitarios abandonen la carrera (Cuadro 1).

Cuadro 1. Estado Actual y Último año cursado. 20102016

\begin{tabular}{lrlr}
\hline $\begin{array}{c}\text { Estado } \\
\text { actual }\end{array}$ & $\begin{array}{c}\text { Porcentaje } \\
\text { válido }\end{array}$ & $\begin{array}{c}\text { Último año } \\
\text { cursado }\end{array}$ & $\begin{array}{c}\text { Porcentaje } \\
\text { válido }\end{array}$ \\
\hline Activo & 28.0 & Primero & 24.0 \\
Desertó & 47.0 & Segundo & 8.0 \\
Egresó & 25.0 & Tercero & 9.0 \\
& & Cuarto & 3.0 \\
& & Quinto & 56.0 \\
\hline \multicolumn{1}{r}{ Total } & $\mathbf{1 0 0 . 0}$ & Total & $\mathbf{1 0 0 . 0}$ \\
\hline
\end{tabular}

Se continuó el estudio tomando en cuenta a los estudiantes activos en la carrera de Ing. en Sistemas de Información. De los cuales el 50\% pertenecen a la cohorte 2012-2016 y el 50\% restante a las dos cohortes anteriores (Cuadro 2). Se realizó un análisis sobre la cantidad de asignaturas aprobadas y reprobadas por cada año de estudio, observándose que a medida que el estudiante avanzó de año académico mejoró su rendimiento académico, ya que en el trayecto del estudio de la carrera aumentó la cantidad de asignaturas aprobadas, disminuyendo así tambiém la cantidad de asignaturas reprobadas (Cuadro 3).

Cuadros 2 y 3. Años de estudio en la carrera y Asignaturas reprobadas por año. Estudiantes Activos.

\begin{tabular}{|c|c|}
\hline Años en la carrera & Porcentaje válido \\
\hline 5 & 50.0 \\
\hline 6 & 28.6 \\
\hline 7 & 21.4 \\
\hline Total & 100.0 \\
\hline
\end{tabular}

Fuente: Secretaría de Facultad. FAREM Matagalpa

\begin{tabular}{|c|c|c|c|c|c|}
\hline $\begin{array}{c}\text { Asignaturas } \\
\text { reprobadas }\end{array}$ & $\begin{array}{l}\text { 1er } \\
\text { año }\end{array}$ & $\begin{array}{l}2 \text { do } \\
\text { año }\end{array}$ & $\begin{array}{l}\text { 3er } \\
\text { año }\end{array}$ & $\begin{array}{l}\text { 4to } \\
\text { año }\end{array}$ & $\begin{array}{l}\text { 5to } \\
\text { año }\end{array}$ \\
\hline 0 & 7 & 15 & 17 & 21 & 26 \\
\hline 1 & 11 & 3 & 6 & 3 & 1 \\
\hline 2 & 3 & 2 & 3 & 1 & 0 \\
\hline $3+$ & 7 & 8 & 2 & 3 & 1 \\
\hline Total & 28 & 28 & 28 & 28 & 28 \\
\hline
\end{tabular}

Fuente: Secretaría de Facultad. FAREM Matagalpa 
De igual manera se analizó el comportamiento de los promedios generales de los estudiantes activos por cada año cursado, observándose una leve mejoría de las calificaciones a medida que el estudiante avanzó de año académico (Figura 8). Lo anterior recalca la importancia de analizar qué factores inciden en el rendimiento académico de los universitarios.
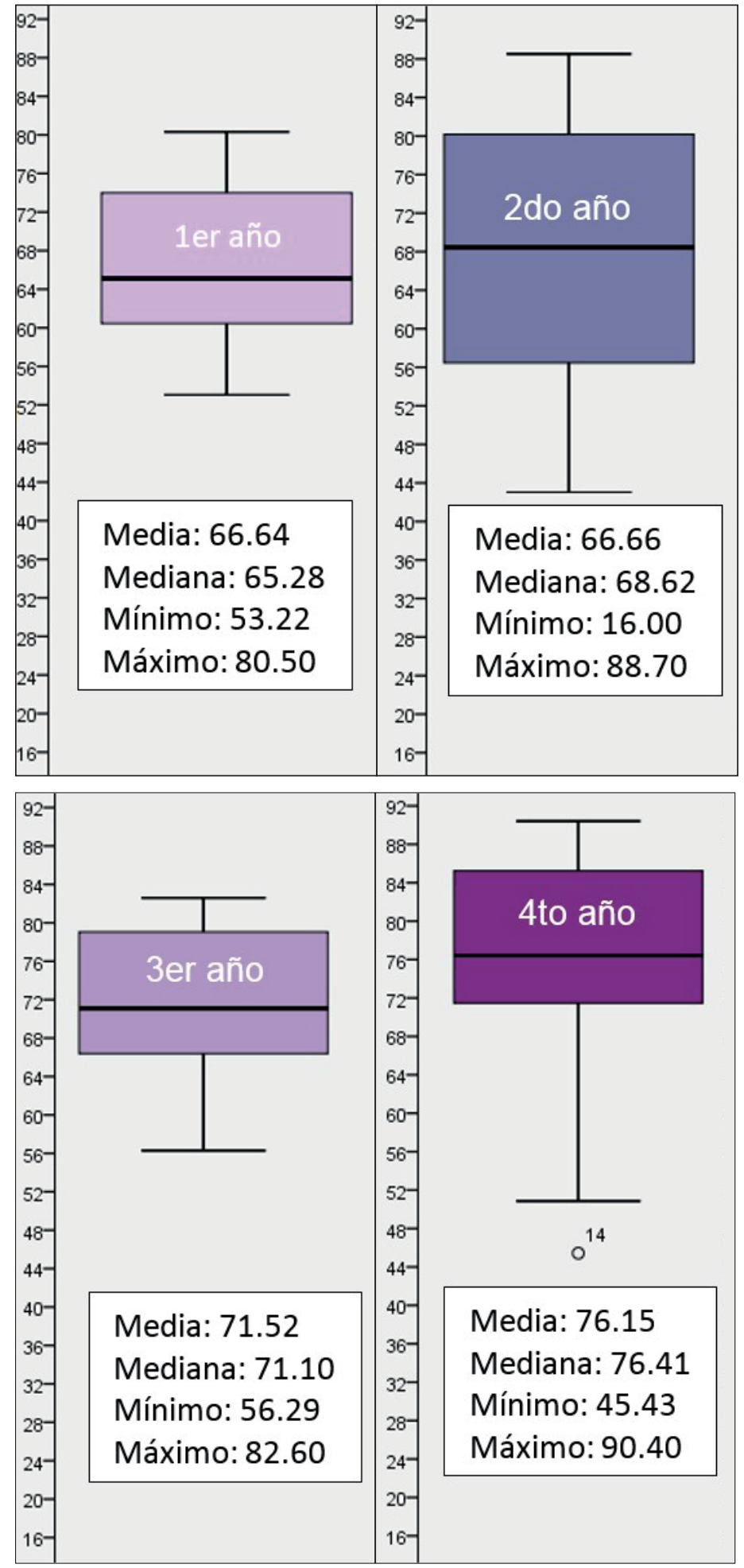

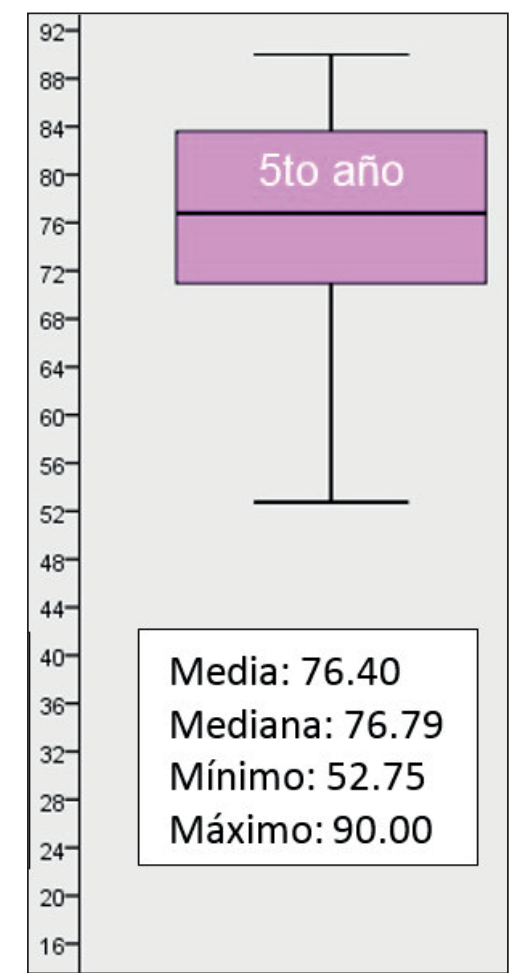

Figura 8. Promedios por Año. Estudiantes Activos

Fuente: propia a partir de datos análisis de datos suministrados por la Secretaría de Facultad. FAREM Matagalpa

\section{Determinantes personales}

Los determinantes personales son aquellos considerados característicos del estudiante, tales como la competencia cognifiva, motivación, condiciones cognitivas, autoconcepto académico, autoeficacia percibida, bienestar psicológico, satisfacción y abandono con respecto a los estudios, asistencia a clase, inteligencia, aptitudes, sexo, formación académica previa a la universidad y la nota de acceso a la misma. (Castejón \& Pérez, 1998); (Garbanzo, 2007); (Miñano, Cantero, \& Castejón, 2008). Se tomaron en cuenta los indicadores de cada factor mencionado, se analizaron $\mathrm{y}$ se formularon preguntas adaptadas al contexto educativo del estudiante encuestado de manera que las comprendiera y las respondiera adecuadamente.

La mayor parte de los estudiantes asumieron que sus resultados académicos son consecuencia de su propio esfuerzo, una minoría confía en su capacidad como estudiante para obtener los resultados deseados (Figura 
9). Todos asumenque el rendimiento académico depende de sí mismos (Figura 10). La mayoría siente fatiga ante la enorme cantidad de trabajos académicos que se les asigna (Figura 11). La asistencia a clase en general es continua (Figura 12). Estos resultados muestran que el esfuerzo, la capacidad y el exceso de tareas académicas son factores causales que los estudiantes reflejan al justificar sus resultados académicos, casi todos los resultados fueron satisfactorios para la mayoría de los estudiantes, con excepción a la cantidad de tareas académicas que se les asigna, esto podría estar disminuyendo la productividad académica de los estudiantes por el poco tiempo que tienen para cumplir dichas asignaciones, también es un factor que cansa y desmotiva al estudiante, por lo que es importante que se dé atención a este problema para buscar acciones que mejoren la situación planteada.

(Garbanzo, 2007), afirma que está demostrado que asumir los resultados académicos se deben a la propia capacidad y esfuerzo y ambos son influyentes en el logro de buenos resultados académicos. La asistencia a clases es un factor influyente para obtener mejores resultados. (Montero \& Villalobos, 2004), aseguran que los estudiantes que asisten continuamente a clase tienen mejores calificaciones que los estudiantes que asisten esporádicamente, de igual forma, creen que el tipo de colegio de donde procede el estudiante es relevante en la universidad.

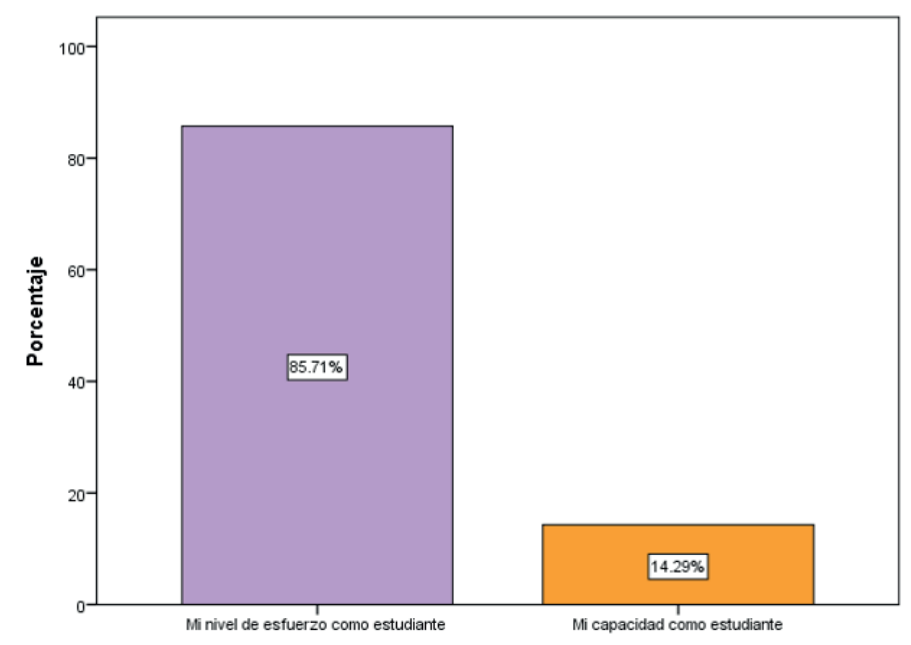

Figura 9. Resultados académicos son consecuencia de

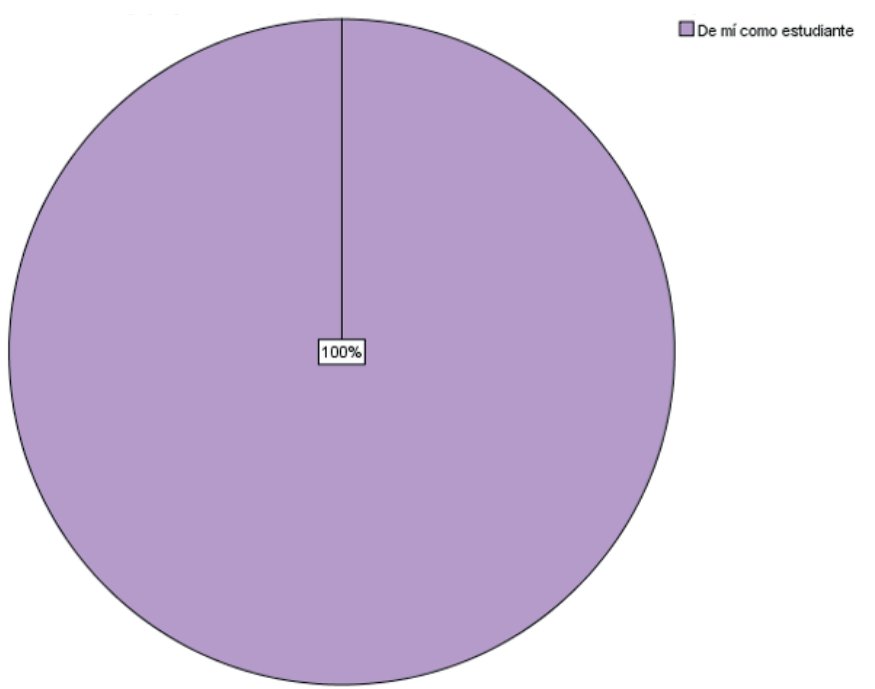

Figura 10. Resultados académicos dependen de

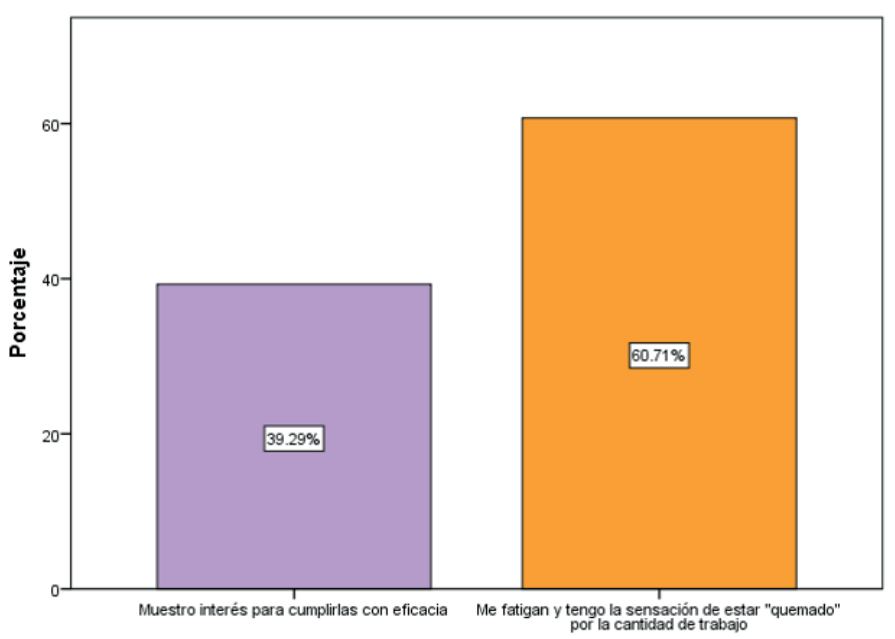

Figura 11. Actitud frente a las actividades académicas

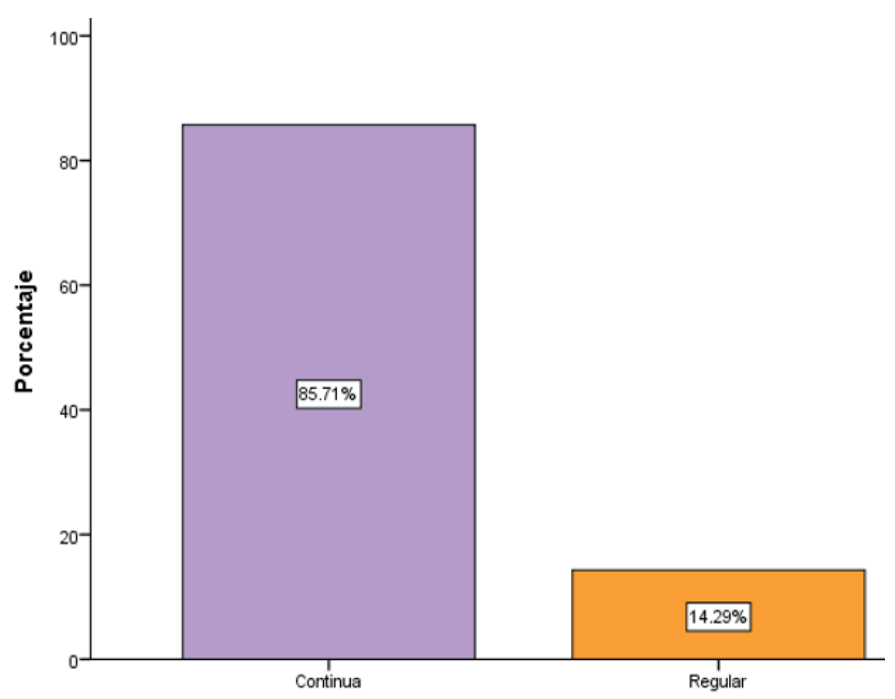

Figura 12. Asistencia a Clase

Fuente: propia a partir de encuesta aplicada a estudiantes 
Se muestran los resultados concernientes a las calificaciones obtenidas por los estudiantes activos en el último año de la secundaria, el examen de admisión y el promedio general obtenido en la universidad (Figura 13), se observan mejores resultados en las notas de la secundaria y los resultados más bajos en el examen de admisión.
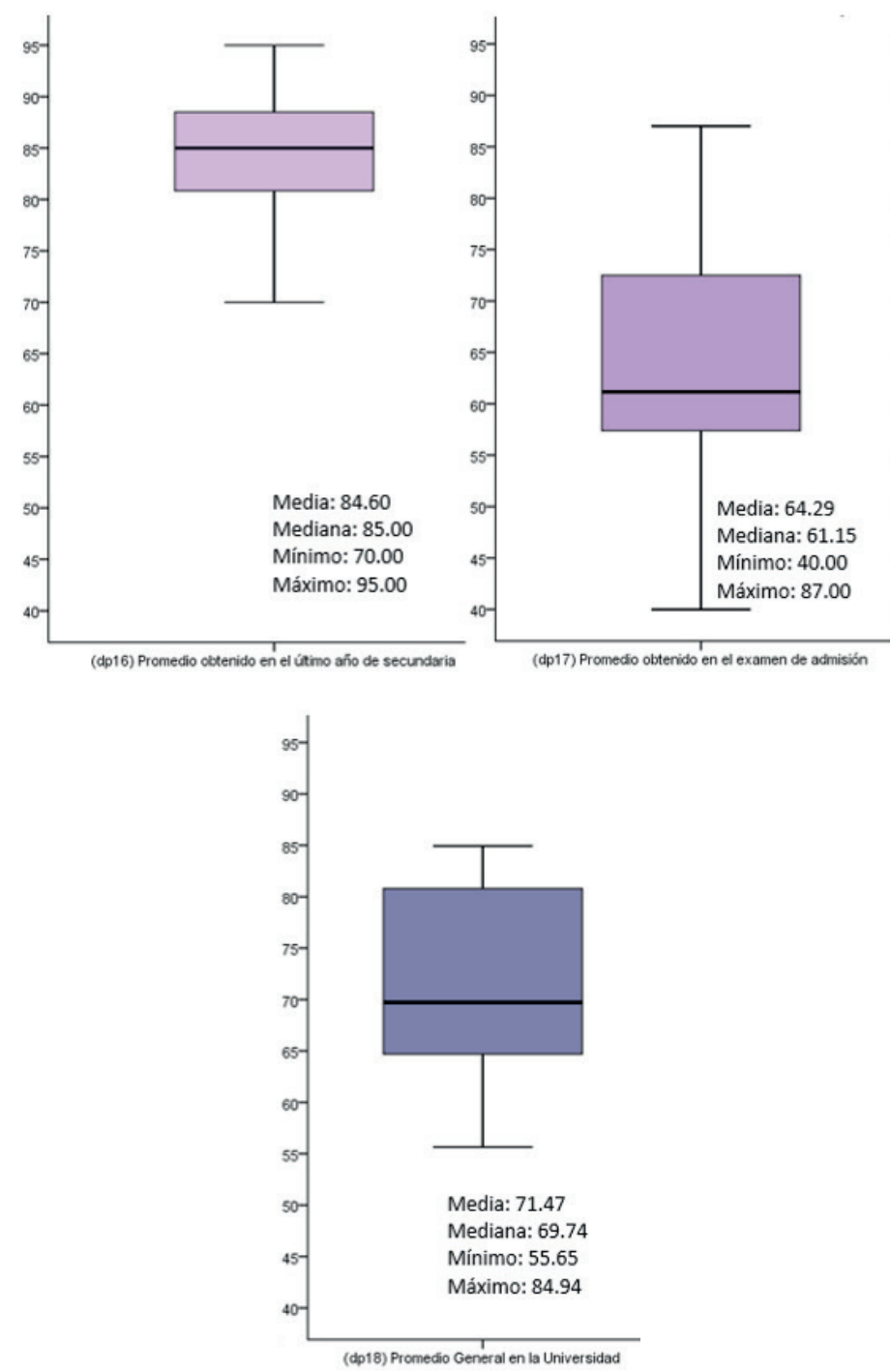

Figura 13. Formación académica Previa y en la Universidad Fuente: propia a partir de encuesta aplicada a estudiantes

(Garbanzo, 2007), señala que las calificaciones obtenidas en secundaria predicen el rendimiento académico del estudiante en la universidad, por otro lado, (Carrión, 2002), menciona que la nota obtenida en el examen de admisión a la universidad es fundamental y es un indicador predictor del rendimiento académico en la universidad. Para verificar tales afirmaciones se realizaron dos análisis de contingencia, el primero para las variables Promedio último año de secundaria (DP16) con Promedio General Universidad (DP18) y el segundo para las variables Nota de Examen de Admisión (DP17) con Promedio General Universidad (DP18).

Se realizó un análisis de correlación de pearson para las variables DP16 y DP18. Para (Pedroza (b), 2015), el análisis de correlación de Pearson permite determinar si hay asociación entre las variables analizadas, donde se toma como referencia un nivel crítico de comparación $\alpha=0.05$.

El análisis de Correlación de Pearson realizado para las variables DP16 y DP18, (Cuadro 4) muestra un coeficiente $r=0.27$, valor próximo a 0 , esto indica una débil asociación entre las variables, obtenido con un $\mathrm{p}=0.1655$, que resulta mayor que el nivel crítico de comparación $\alpha=0.05$, valor estadístico para una correlación NS, por lo tanto, se demostró que no existe correlación entre las variables DP16 y DP18, por ello, se rechaza la hipótesis señalada por Garbanzo (2007), que asegura que el promedio del último año de secundaria predice el rendimiento académico en la universidad.

Cuadro 4. Años de estudio en la carrera y Asignaturas reprobadas por año. Estudiantes Activos.

\begin{tabular}{lllrr} 
Variable (1) & Variable (2) & $\mathrm{n}$ & Pearson & p-valor \\
\hline DP16 & DP16 & 28 & 1.00 & $<0.0001$ \\
DP16 & DP18 & 28 & 0.27 & 0.1655 \\
& & & & \\
DP18 & DP16 & 28 & 0.27 & 0.1655 \\
DP18 & DP18 & 28 & 1.00 & $<0.0001$ \\
\hline
\end{tabular}

Fuente: propia a partir de encuesta aplicada a estudiantes

Se realizó un análisis de regresión lineal para las variables DP16 y DP18. Pedroza (2015), afirma que este análisis se realiza para cuantificar el grado de asociación entre dos variables cuantitativas continuas, dicho grado se mide estadísticamente por el Coeficiente de Regresión "b" y se realiza bajo la hipótesis nula de 
$\mathrm{H}_{0}: \beta=0$; este análisis supone la existencia de una relación funcional, del tipo lineal en sus parámetros entre una variable respuesta y una o más variables predictoras o regresoras.

El análisis de regresión realizado para las variables DP16 y DP18, (Cuadro 5), resultó con un coeficiente de regresión " $b$ " igual a 0.23 , con un $p=0,1754$, que es mayor que el nivel crítico de comparación $\alpha=0.05$. Por lo tanto, no se rechaza la hipótesis nula de Ho: $\beta=$ 0 , así, la respuesta estadística obtenida es una regresión No Significativa, por lo que se demostró que no existe relación cuantitativa entre las variables DP16 y DP18 como lo aseguraba (Carrión, 2002).

Cuadro 5. Análisis de Regresión Lineal. Nota de Examen de Admisión \& Promedio Universidad.
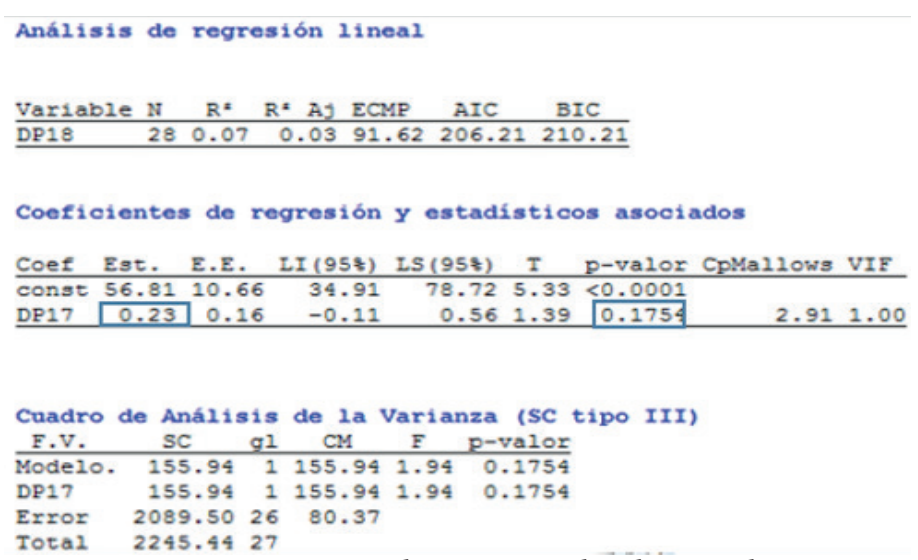

Fuente: propia a partir de encuesta aplicada a estudiantes

Un factor importante de analizar es la capacidad del estudiante para adaptarse al trabajo de grupo. Para (Pedroza (b), 2015), el grado de asociación que ocurre entre dos variables cualitativas ordinales, se mide mediante el Coeficiente de Correlación de Tau C de Kendall. Se realizó un análisis de contingencia mediante la prueba Tau C de Kendall, entre sector donde vive el estudiante (DG5) y su buena adaptación para trabajar en grupo (DP14). La Cuadro de contingencia (Cuadro 6) muestra total acuerdo en la mayoría de los estudiantes, independientemente del sector donde vive, únicamente dos estudiantes de la zona urbana muestran problemas de adaptación para trabajar en grupo.
Cuadro 6. Tabla de Contingencia (DG5) Sector donde vive * (DP14) Considera usted que se adapta muy bien para trabajar en grupo

\begin{tabular}{|c|c|c|c|c|c|c|}
\hline & \multicolumn{3}{|c|}{$\begin{array}{l}\text { (dp14) considera usted que se } \\
\text { adapta muy bien para trabajar en } \\
\text { grupo }\end{array}$} & \multirow[t]{2}{*}{ Total } \\
\hline & & & $\begin{array}{c}\text { En } \\
\text { desacuerdo }\end{array}$ & $\begin{array}{c}\text { De } \\
\text { acuerdo }\end{array}$ & $\begin{array}{l}\text { Totalmente } \\
\text { de acuerdo }\end{array}$ & \\
\hline \multirow{6}{*}{$\begin{array}{l}(\mathrm{dg} 5) \\
\text { Sector } \\
\text { donde } \\
\text { vive }\end{array}$} & \multirow{2}{*}{$\begin{array}{l}\text { Zona } \\
\text { rural }\end{array}$} & Recuento & 0 & 2 & 4 & 6 \\
\hline & & $\%$ del total & $0.0 \%$ & $7.1 \%$ & 14.3 & $21.4 \%$ \\
\hline & \multirow{2}{*}{$\begin{array}{l}\text { Zona } \\
\text { urbana }\end{array}$} & Recuento & 2 & 4 & 7 & 13 \\
\hline & & $\%$ del total & $7.1 \%$ & 14.3 & $25.0 \%$ & $46.4 \%$ \\
\hline & \multirow{2}{*}{$\begin{array}{l}\text { Zona } \\
\text { periférica }\end{array}$} & Recuento & 0 & 0 & 9 & 9 \\
\hline & & $\%$ del total & $0.0 \%$ & $0.0 \%$ & $32.1 \%$ & $32.1 \%$ \\
\hline \multirow[t]{2}{*}{ Total } & & Recuento & 2 & 6 & 20 & 28 \\
\hline & & $\%$ del total & $7.1 \%$ & $21.4 \%$ & $71.4 \%$ & $100.0 \%$ \\
\hline
\end{tabular}

Fuente: propia a partir de encuesta aplicada a estudiantes

El análisis de Correlación de Tau C de Kendall realizado para las variables DG5 y DP14, (Cuadro 7) muestra un coeficiente $r=0.22$, con un $p=0.038$, que resulta menor que el nivel crítico de comparación $\alpha=0.05$, valor estadístico para una correlación Significativa $(*)$, por lo tanto, se demostró que existe correlación entre las variables DG5 y DP14, por ello, se confirma la hipótesis de que hay asociación entre el sector donde viven los estudiantes y la adaptación para trabajar en grupo.

Cuadro 7. Correlación entre DG5 y DP14

\begin{tabular}{lccrl}
\hline & Valor & $\begin{array}{l}\text { Error tip. } \\
\text { asint* }\end{array}$ & $\begin{array}{l}\text { T } \\
\text { aproximada }\end{array}$ & $\begin{array}{l}\text { Sig. } \\
\text { aproximada }\end{array}$ \\
\hline $\begin{array}{l}\text { Ordinal por } \\
\text { ordinal Tau-c de }\end{array}$ & .222 & .107 & 2.080 & .038 \\
$\begin{array}{l}\text { Kendall } \\
\text { N de casos }\end{array}$ & & & & \\
validos & 28 & & & \\
\hline
\end{tabular}

a. Asumiendo la Hipótesis alternativa

b. empleando el error típico asitóntico basado basado en la hipótesis nula

Fuente: propia a partir de encuesta aplicada a estudiantes

Se encontraron resultados relevantes con respecto a algunos factores personales; tales como que la mayoría de los estudiantes consideran ser motivados constantemente por sus compañeros para seguir estudiando (Figura 14). Una minoría de los estudiantes han tenido afectación en su rendimiento académico debido a problemas emocionales (Figura 15). Esto 
indica que la motivación es un factor determinante en el rendimiento académico.

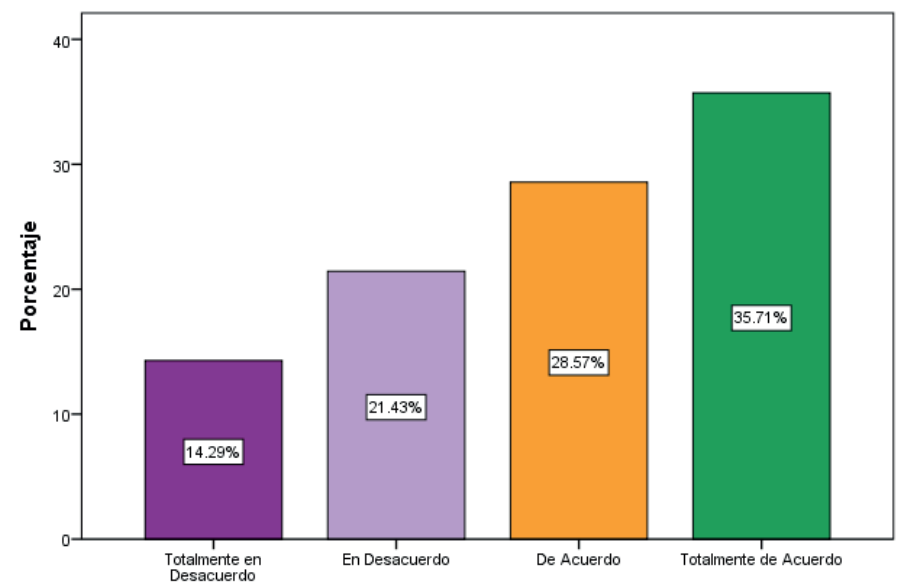

Figura 14. Motivación de los compañeros

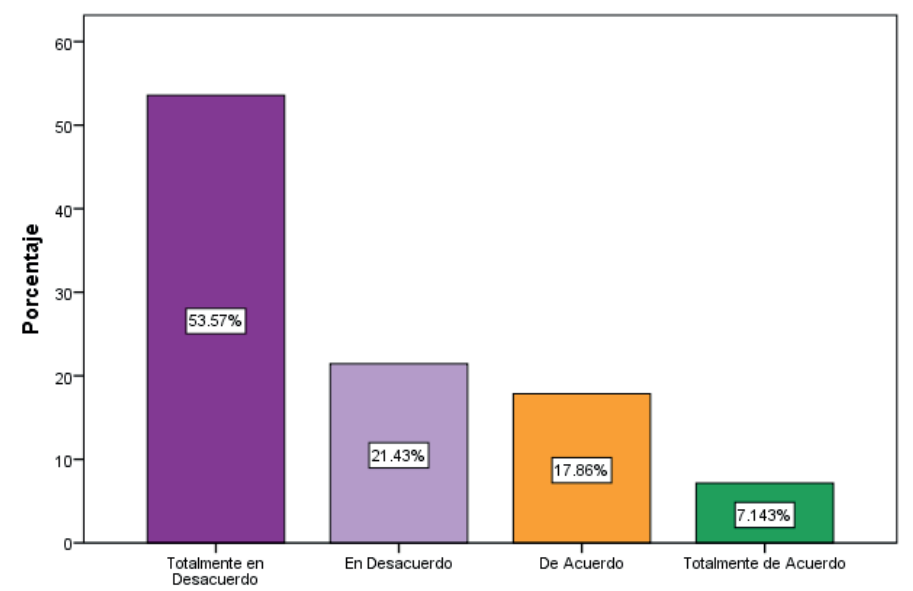

Figura 15. Rendimiento académico ha sido afectado por problemas emocionales

Para ver el comportamiento de algunos factores se realizó un análisis de componentes principales, que para (Pedroza (b), 2015), son combinaciones lineales que tienen la máxima variabilidad posible, uno independiente del otro. El criterio de clasificación utilizado fue el sexo de los estudiantes y las variables combinadas fueron los determinantes personales de valoración (Figura 16).

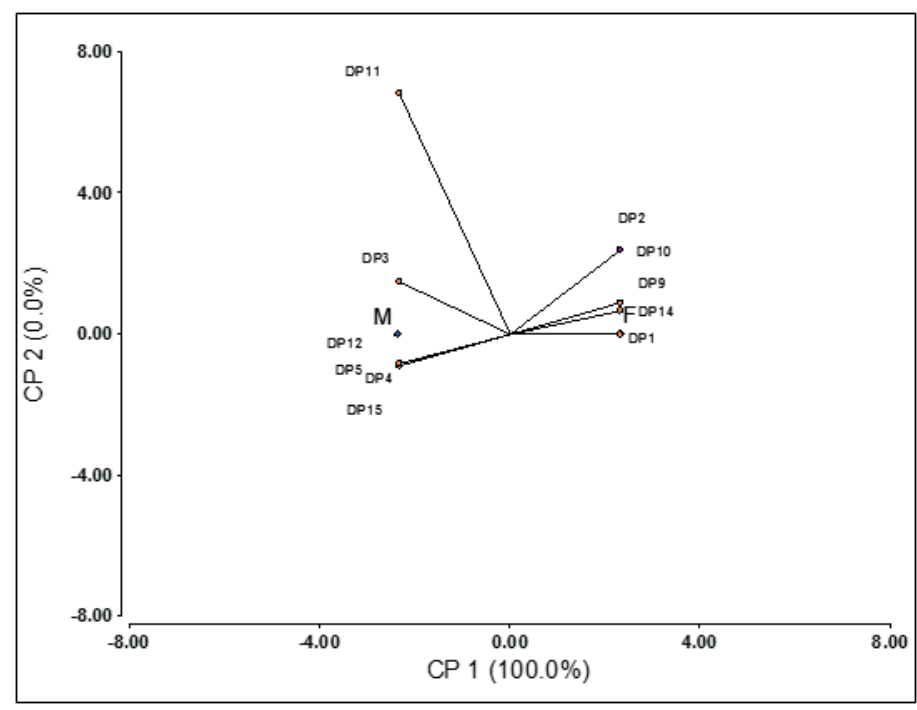

Figura 16. Otros Determinantes Personales clasificados por sexo Fuente: propia a partir de encuesta aplicada a estudiantes

Se observó que más varones seleccionaron las variables DP3, DP4, DP5, DP12 y DP15, que se refieren a factores personales como motivación, bienestar psicológico e inteligencia emocional. La mayoría de ellos determinaron que cada actividad académica asignada es tomada como un reto, sienten orgullo por estudiar en la FAREM Matagalpa de la UNAN Managua, son motivados constantemente por sus compañeros, aumenta su autoestima cuando obtienen el rendimiento académico que esperaban y han sido afectados académicamente cuando tienen problemas emocionales.

Por otro lado, más mujeres seleccionaron las variables DP1, DP2, DP9, DP10 y DP14, que se refieren a competencia cognitiva, bienestar psicológico e inteligencia emocional. Las estudiantes opinaron que tienen capacidad y habilidad para cursar y culminar la carrera con éxito, su rendimiento académico mejora cada semana, muestran satisfacción y felicidad por sus estudios y se adaptan con facilidad a trabajar en grupo. La variable DP11 no fue seleccionada como aceptada en ninguno de los sexos, y es que ningún estudiante ha pensado en la posibilidad de abandonar la carrera. 


\section{Determinantes sociales}

(Torres \& Rodríguez, 2006), opinan que los determinantes sociales determinada por el ingreso familiar, la escolaridad de la madre y del padre, el tipo y ubicación de la vivienda, ha sido reportada como una variable relacionada con el hecho de que los estudiantes sufran fracasos escolares o repitan cursos. Por ello, se hizo hincapié en conocer la situación de los estudiantes con respecto a factores sociales como el entorno familiar, nivel educativo de los padres, la cultura, contexto socioeconómico, apoyo familiar, entre otros factores relevantes. Para (Meneses, Morillo, Navia, \& Grisales, 2013), "la familia sigue siendo un escenario de socialización y de formación muy importante, esto permite que el individuo se desenvuelva de manera apropiada en la sociedad" (p. 443).

Los resultados muestran que fue decisión propia la elección de la carrera estudiada, una minoría fue por decisión familiar; el ambiente familiar de convivencia es democrático para muchos, pero hay estudiantes que viven en un ambiente autoritario (Cuadro 6). Los padres de la mitad de los estudiantes tienen educación primaria, y las madres muestran el mismo porcentaje de educación primaria y secundaria (Cuadro 7).

Cuadro 6. Entorno Familiar

\begin{tabular}{ll}
\hline Elección de la carrera (\%) & Ambiente familiar (\%) \\
\hline Propia: $96.4 \%$ & Democrático: 89.3 \\
Familiar: $3.6 \%$ & Autoritario: 7.1 \\
& No convivo con la familia: \\
& 3.6 \\
\hline Total: 100 & Total: 100 \\
\hline
\end{tabular}

Fuente: propia a partir de encuesta aplicada a estudiantes

Cuadro 7. Nivel Educativo de los Padres

\begin{tabular}{lrr}
\hline Nivel educativo & \multicolumn{1}{c}{ Padre (\%) } & Madre (\%) \\
\hline Analfabeto & 7.1 & 3.6 \\
Primaria & 50.0 & 42.9 \\
Secundaria & 17.9 & 42.9 \\
Universitario & 21.4 & 10.7
\end{tabular}

\begin{tabular}{lrr} 
Posgraduado & 3.6 & 00.0 \\
\hline Total & 100.0 & 100.0 \\
\hline
\end{tabular}

Fuente: propia a partir de encuesta aplicada a estudiantes

(Garbanzo, 2007), explica que el capital cultural se refiere a las relaciones entre el ambiente familiar y los recursos didácticos a los que los estudiantes acuden, de manera que enriquezcan el ambiente educativo y contribuya a resultados académicos positivos. La mayoría mostró interés en ampliar sus conocimientos usando internet, y la minoría por medio de relaciones familiares marcadas por discusiones que propician el saber (Figura 17). (Lozano, 2004), la condición educativa atribuida a la familia está fuera de toda duda y discusión, siendo muy importante el papel de los padres en el progreso y desarrollo educativo de sus hijos, la motivación del logro depende también del nivel cultural de los padres, sin embargo, en este estudio, se observa que la familia no contribuye mucho al desarrollo del capital cultural del estudiante.

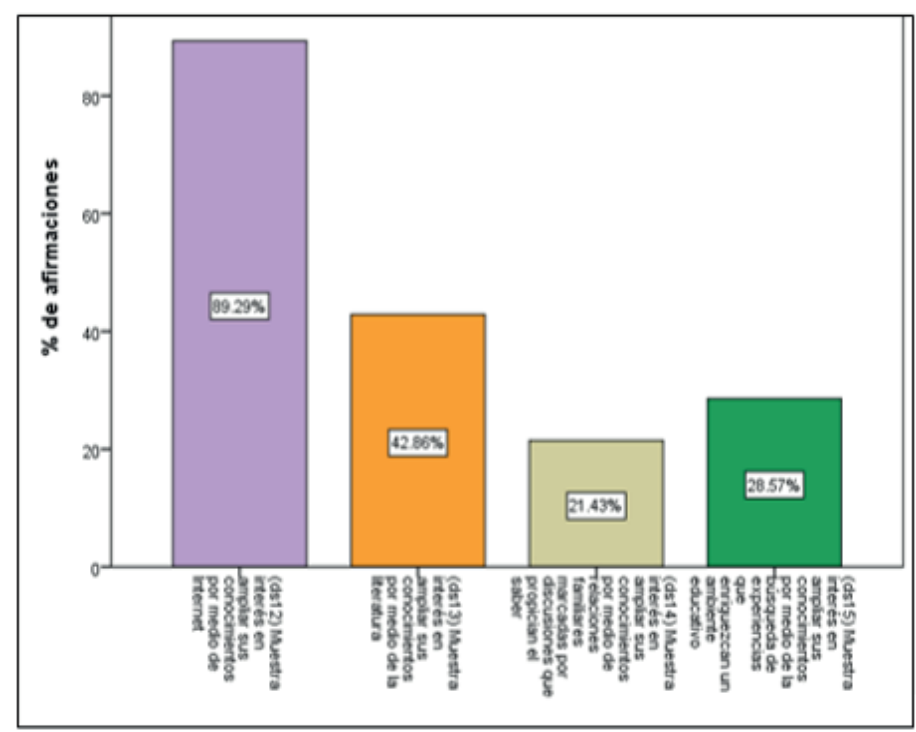

Figura 17. Capital Cultural

Fuente: propia a partir de encuesta aplicada a estudiantes

Se estudiaron algunos indicadores concernientes al contexto socioeconómico del estudiante (Figura 18), en promedio, los estudiantes cuentan alrededor de C\$1,289.29 al mes para sus gastos, entre 5 y 6 personas habitan en la misma vivienda, en promedio, 2 de ellas contribuyen a generar ingresos en el hogar; esto muestra 
que hay pocos ingresos para el estudiante debido a cantidad de personas que sostienen económicamente el hogar; cabe recalcar que hay casos donde viven hasta 11 personas, y casos donde no hay ingreso monetario para el estudiante.
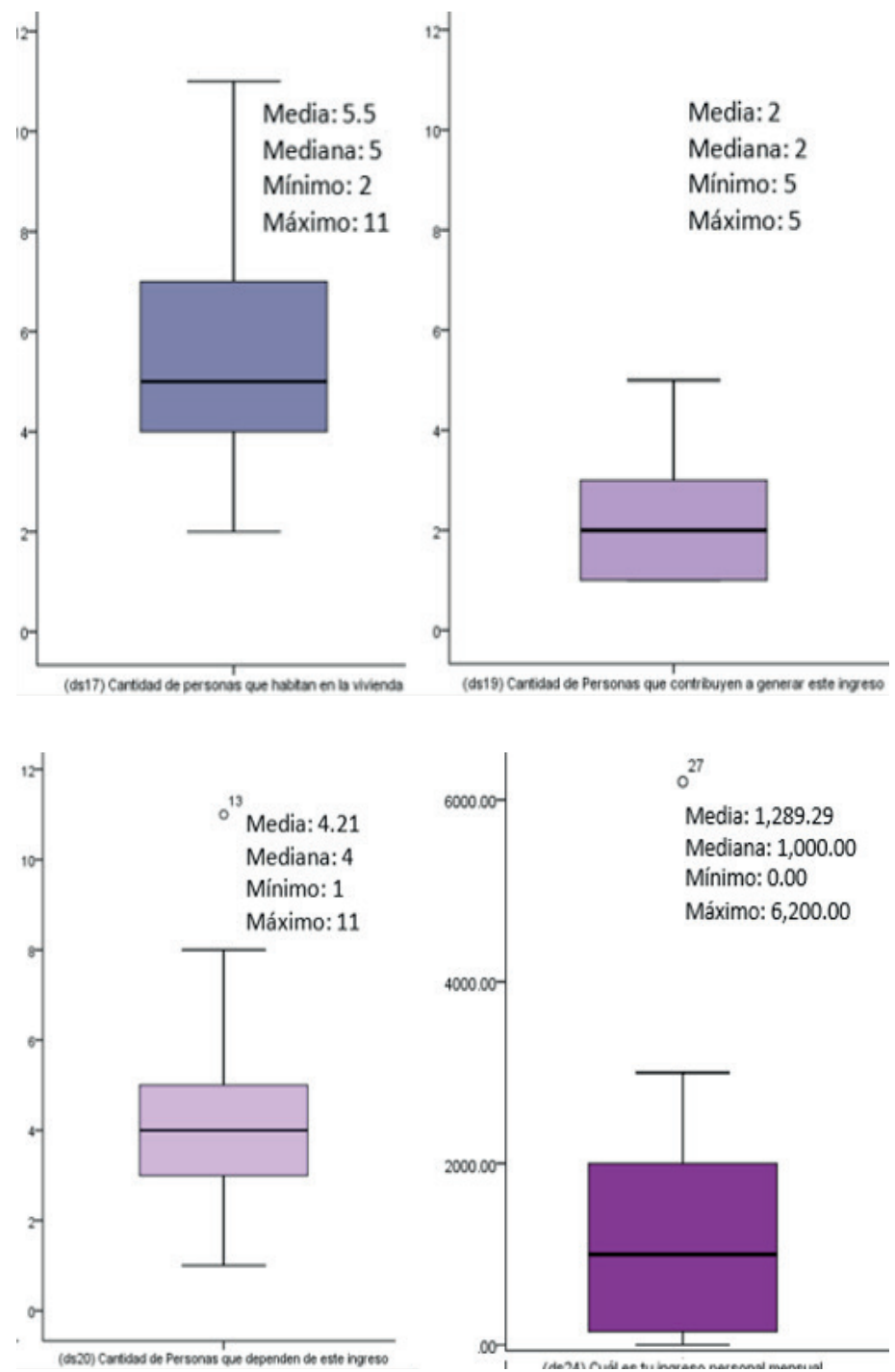

Figura 18. Contexto Socioeconómico Cultural

Fuente: propia a partir de encuesta aplicada a estudiantes

Se realizó un análisis de correlación de Pearson para las variables Promedio General en la Universidad (DP18) y el ingreso personal mensual del estudiante (DS24) (Tabla 8), el resultado muestra un coeficiente $\mathrm{r}=$ 0.43 con un $p=0.0209$, que resulta menor que el nivel crítico de comparación $\alpha=0.05$, valor estadístico para una correlación significativa $(*)$, por lo tanto, existe correlación entre las variables DP18 y DS24, así se confirma que hay asociación entre el ingreso personal mensual del estudiante y el promedio general obtenido en la universidad.

Cuadro 8. Correlación de Pearson.

\begin{tabular}{lllll}
\hline Variable (1) & Variable (2) & $\mathbf{n}$ & Pearson & p-valor \\
\hline DP18 & DP18 & 28 & 1.00 & $<0.0001$ \\
DP18 & DS24 & 28 & -0.43 & 0.0209 \\
& & & & \\
DS24 & DP18 & 28 & -0.43 & 0.0209 \\
DS24 & DS24 & 28 & 1.00 & $<0.0001$ \\
\hline
\end{tabular}

Fuente: propia a partir de encuesta aplicada a estudiantes

Se obtuvieron otros datos relevantes concernientes al factor económico (Figura 19), la mitad de los estudiantes perciben en su hogar un ingreso entre $\mathrm{C} \$ 3,500.00$ y $\mathrm{C} \$ 10,000.00$, un $21.43 \%$ de los mismos perciben menos de $\mathrm{C} \$ 3,500.00$, siendo éstos ingresos bajos para mantener un hogar. Por otro lado, la mayor parte de los estudiantes negó haber obtenido mal rendimiento académico por problemas económicos (Figura 20).

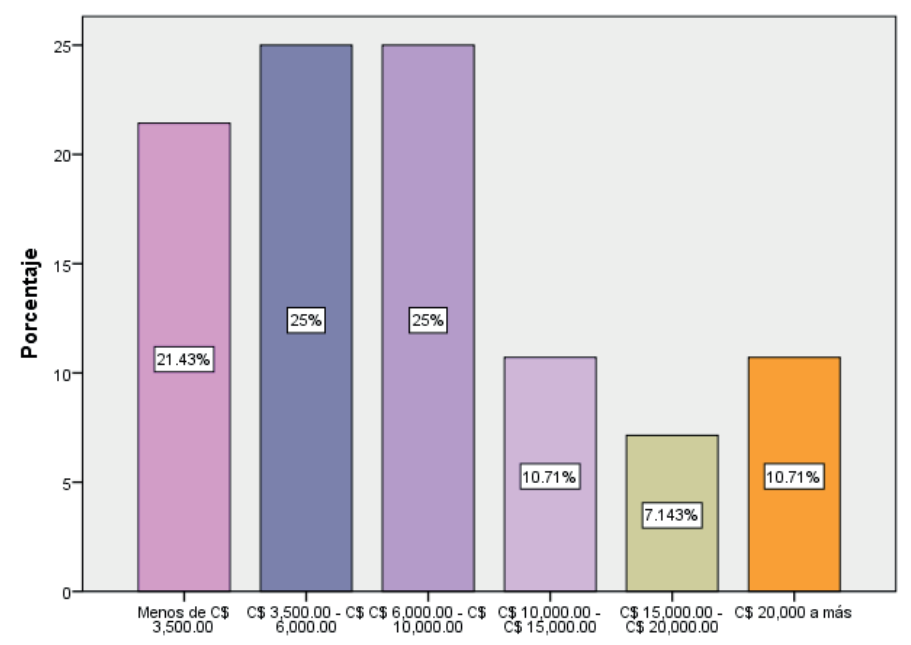

Figura 19. Ingreso Mensual Promedio en el hogar 


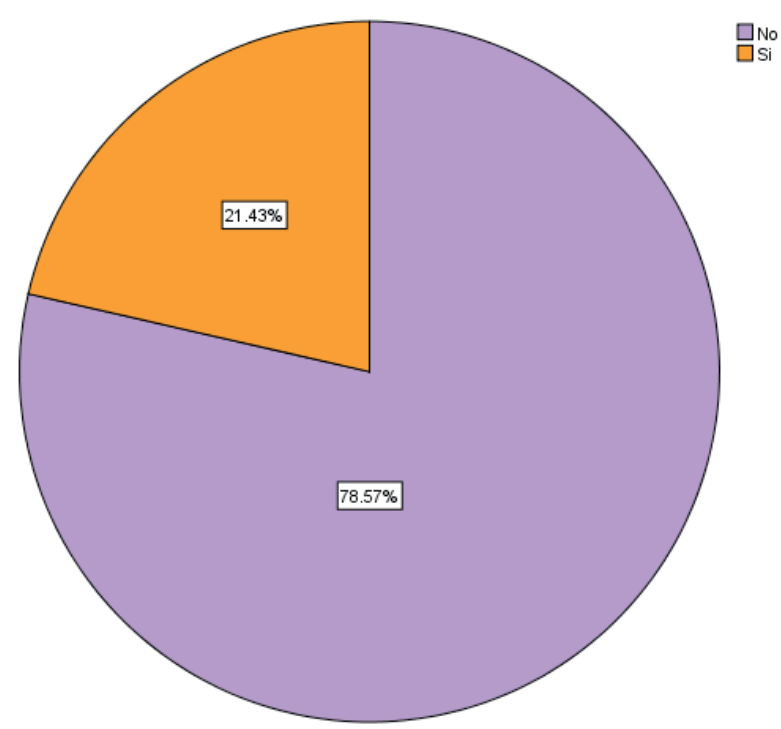

Figura 20. Existencia de un mal rendimiento académico debido a problemas económicos
Se realizó un análisis de contingencia (Cuadro 9), donde se observa que los estudiantes donde el ingreso es menor a $\mathrm{C} \$ 10,000.00$ se fatigan por la cantidad de trabajo que se les asigna en las diversas clases de la carrera. El análisis de Correlación de Tau C de Kendall realizado para la variable que valoran su opinión sobre las actividades académicas (DP8) y el ingreso mensual promedio en el hogar (DS18) (Cuadro 10), muestra un coeficiente $r=-0.383$, con un $p=0.043$, que resulta menor que el nivel crítico de comparación $\alpha=0.05$, valor estadístico para una correlación Significativa (*), por lo tanto, se demostró que hay asociación entre la valoración de las actividades académicas y el ingreso mensual promedio en el hogar.

\begin{tabular}{|c|c|c|c|c|c|c|c|c|c|}
\hline & & & \multicolumn{6}{|c|}{ (ds18) Ingreso mensual promedio en tu hogar } & \multirow[t]{2}{*}{ Total } \\
\hline & & & $\begin{array}{c}\text { Menos } \\
\text { de CS } \\
\mathbf{3 , 5 0 0 . 0 0}\end{array}$ & $\begin{array}{c}C \$ \\
3,500.00 \\
-C \$ \\
6,000.00\end{array}$ & $\begin{array}{c}C \$ \\
6,000.00 \\
-C \$ \\
10,000.00\end{array}$ & $\begin{array}{c}\mathrm{C} \$ \\
10,000.00 \\
-\mathrm{C} \$ \\
15,000.00\end{array}$ & $\begin{array}{c}C \$ \\
15,000.00 \\
-C \$ \\
20,000.00\end{array}$ & $\begin{array}{c}\text { CS } \\
\text { 20,000.00 } \\
\text { a más }\end{array}$ & \\
\hline \multirow{4}{*}{$\begin{array}{l}(\mathrm{dp} 8) \text { En } \\
\text { cuanto a las } \\
\text { actividades } \\
\text { academicas }\end{array}$} & Muestro & Recuento & 1 & 2 & 3 & 2 & 1 & 2 & 11 \\
\hline & $\begin{array}{l}\text { interes para } \\
\text { cumplirlas } \\
\text { con efiucacia }\end{array}$ & $\%$ del total & $3.6 \%$ & $7.1 \%$ & $10.7 \%$ & $7.1 \%$ & $3.6 \%$ & $7.1 \%$ & $39.3 \%$ \\
\hline & Me fatigan & Recuento & 5 & 5 & 4 & 1 & 1 & 1 & 17 \\
\hline & $\begin{array}{l}\text { y tengo la } \\
\text { sensación } \\
\text { de estar } \\
\text { "quemado" } \\
\text { por la } \\
\text { cantidad de } \\
\text { trabajo }\end{array}$ & $\%$ del total & $17.9 \%$ & $17.9 \%$ & $14.3 \%$ & $3.6 \%$ & $3.6 \%$ & $3.6 \%$ & $60.7 \%$ \\
\hline \multirow[t]{2}{*}{ Total } & & Recuento & 6 & 7 & 7 & 3 & 2 & 3 & 28 \\
\hline & & $\%$ del total & $21.4 \%$ & $25.0 \%$ & $25.0 \%$ & $10.7 \%$ & $7.1 \%$ & $10.7 \%$ & $100 \%$ \\
\hline
\end{tabular}

Cuadro 10. Análisis de Correlación de Tau C de Kendall. DP8 y DS18

\begin{tabular}{lccrl}
\hline & Valor & $\begin{array}{l}\text { Error tip. } \\
\text { asint* }\end{array}$ & $\begin{array}{l}\text { T } \\
\text { aproximada }\end{array}$ & $\begin{array}{l}\text { Sig. } \\
\text { aproximada }\end{array}$ \\
\hline $\begin{array}{l}\text { Ordinal por } \\
\text { ordinal Tau-c de }\end{array}$ & -.383 & .189 & -2.020 & .043 \\
$\begin{array}{l}\text { Kendall } \\
\text { N de casos }\end{array}$ & 28 & & & \\
válidos & & & & \\
\hline
\end{tabular}

a. Asumiendo la Hipótesis alternativa

b. empleando el error típico asitóntico basado basado en la hipótesis nula

Fuente: propia a partir de encuesta aplicada a estudiantes
Otros resultados que destacan con respecto a los determinantes sociales es que existe mayor apoyo de la madre en las tareas académicas que del padre (Figuras 21 y 22). Esto indica que es la madre quien insiste y apoya a sus hijos a que realicen las actividades académicas pertinentes para que los mismos tengan buenos resultados, a la vez que la mayoría de los padres exigen a sus hijos un rendimiento académico con calidad (Figura 23) 


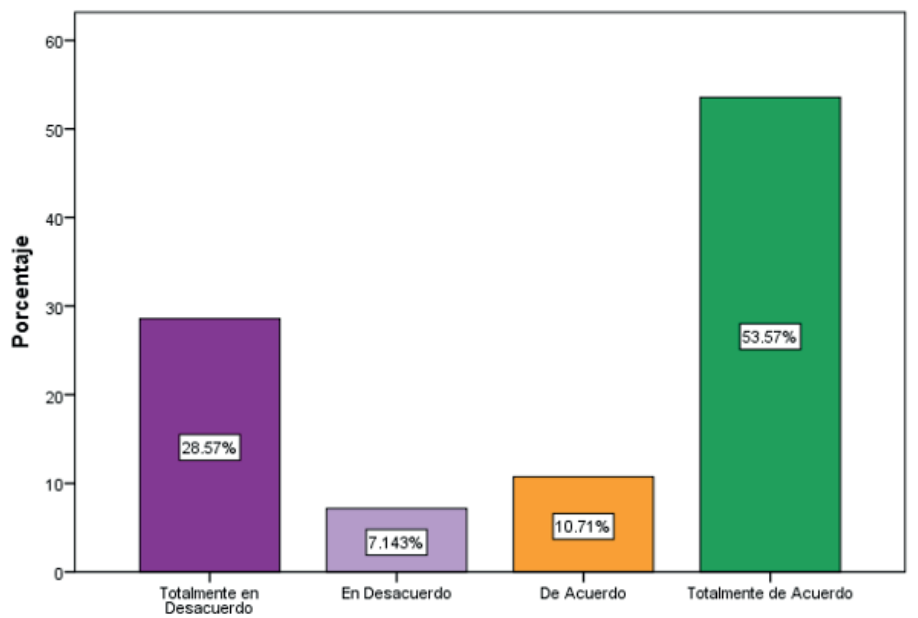

Figura 21. Motivación y Apoyo del Padre

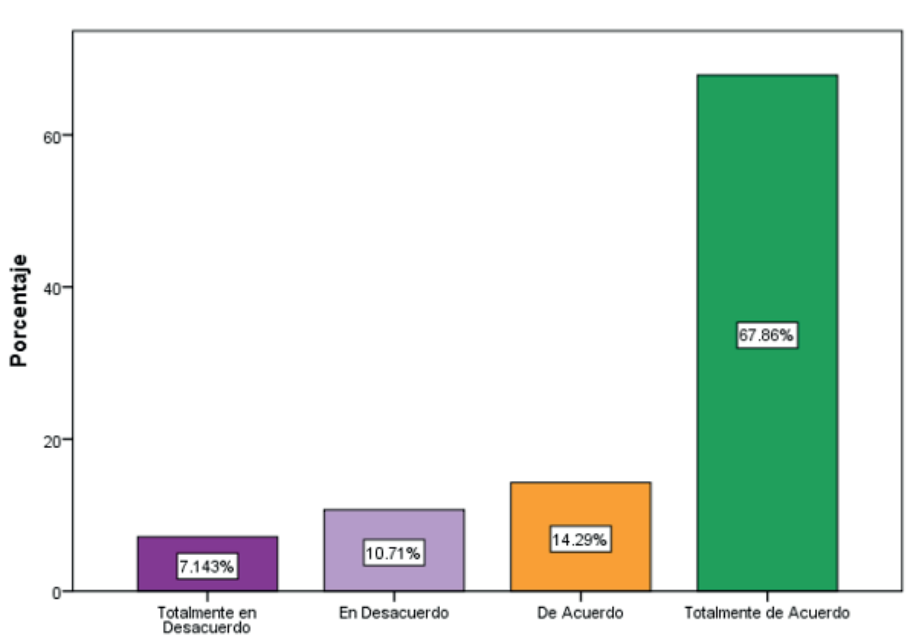

Figura 22. Motivación y Apoyo de la Madre

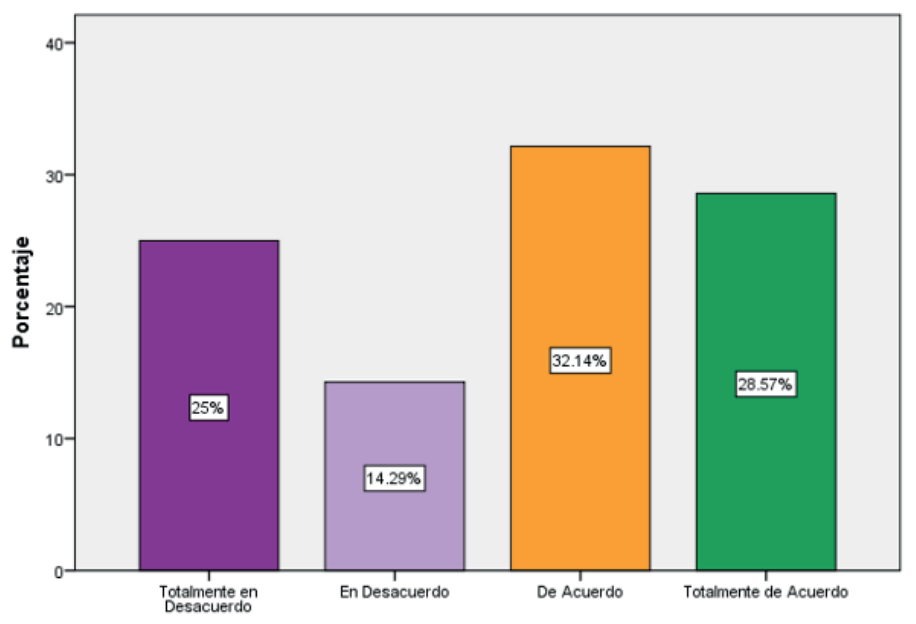

Figura 23. Exigencia de los Padres

Fuente: propia a partir de encuesta aplicada a estudiantes
Algunos Determinantes Sociales se valoraron mediante un análisis de Componentes Principales (ACP), donde el criterio de clasificación utilizado fue sexo y variables combinadas fueron los determinantes sociales con criterios de valoración (Figura 24). Se observó que más varones seleccionaron las variables DS10 y DS11, que se refieren a factores sociales relacionados con el nivel educativo de los padres; tales como que los padres exigen un rendimiento académico de calidad a sus hijos y la motivación y apoyo por parte de la madre en el estudio de los estudiantes.

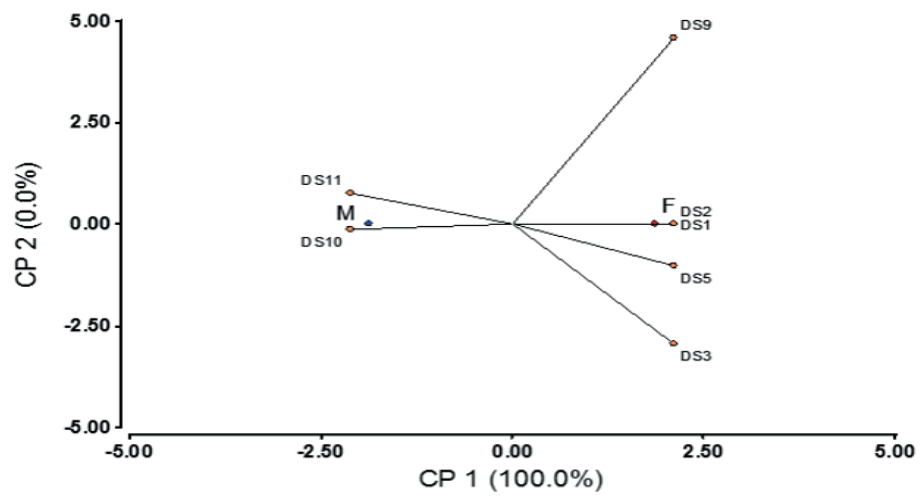

Figura 23. Exigencia de los Padres

Fuente: propia a partir de encuesta aplicada a estudiantes

Por otro lado, las mujeres estuvieron más identificadas con las variables DS1, DS2, DS3, DS5 Y DS9; referidas a determinantes sociales relacionados con las diferencias sociales; donde determinaron un trato igualitario sin distingo de género, raza y clases sociales. Además estuvieron de acuerdo en que la familia ha mostrado interés en el bienestar económico de sus hijos. Casi la mitad de estudiantes expusieron no ser motivados y apoyados por la figura paterna.

\section{Determinantes Institucionales}

Para (Carrión, 2002), los determinantes institucionales son componentes no personales que intervienen en el proceso educativo; tales como metodología docente, horario de clase, tamaño de grupo de clase, entre otros..., se considera de importancia porque tiene una relación directa con el entorno educativo donde el estudiante se desempeña en su quehacer académico. 
(Ocaña, 2011), afirma que el rendimiento académico en la universidad se incrementa si mejora la calidad de la infraestructura donde el estudiante realiza su trabajo académico, tales como comodidades para el estudio, biblioteca, aulas, entre otros, de igual forma opina que el apoyo de profesores tutores y compañeros monitores también es fundamental para mejorar el desempeño de los estudiantes.

Algunos de los indicadores valorados como determinantes institucionales fueron la forma en que el estudiante ingresó a la carrera de Ing. en Sistemas de Información, casi todos los estudiantes clasificaron en primera opción (Figura 25), factor muy importante, que podría determinar que el estudiante está apto para desarrollarse con éxito en el transcurso de sus estudios. La mayoría presentó dificultad para entender y estudiar algunas asignaturas (Figura 26); para una parte de los estudiantes los laboratorios están en condiciones óptimas para desarrollar las clases, pero una cantidad casi similar opina lo contrario (Figura 27).

La mayoría opina que los docentes utilizan una metodología adecuada, que ha ayudado a desarrollar un aprendizaje significativo (Figura 28); un poco más de la mitad afirma que el sistema de becas en la universidad no es equitativo (Figura 29); Para muchos, la biblioteca ofrece excelentes servicios de préstamo de material didáctico (Figura 30); la mayor parte de los estudiantes considera que no se cuenta con buena atención médica y psicológica en la FAREM Matagalpa de la UNAN Managua (Figuras 31 y 32); un poco más de la mitad opina que hay un ambiente estudiantil con apoyo social cuando se presentan problemas serios con algún estudiante (Figura 33) y la gran mayoría afirma que se debe aplicar un examen de admisión para optar al estudio en la FAREM Matagalpa de la UNAN Managua (Figura 34).

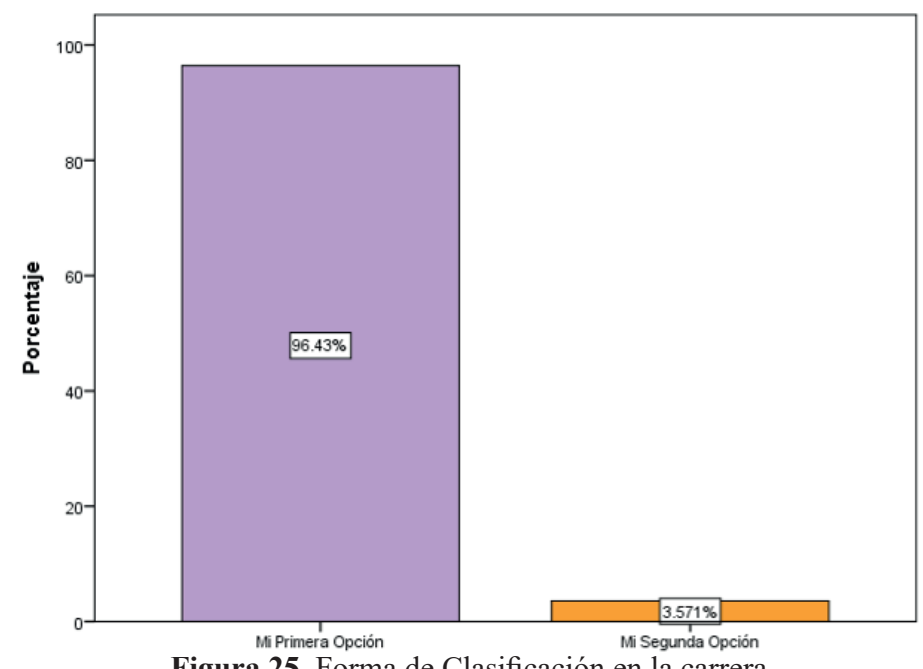

Figura 25. Forma de Clasificación en la carrera

Fuente: propia a partir de encuesta aplicada a estudiantes

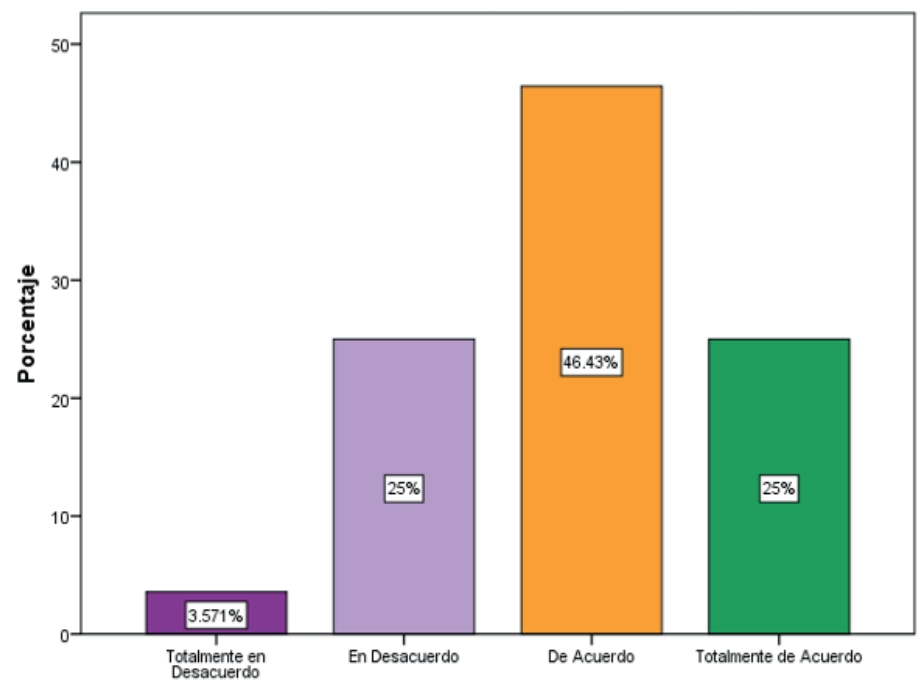

Figura 26. Dificultad para entender y estudiar algunas asignaturas Fuente: propia a partir de encuesta aplicada a estudiantes

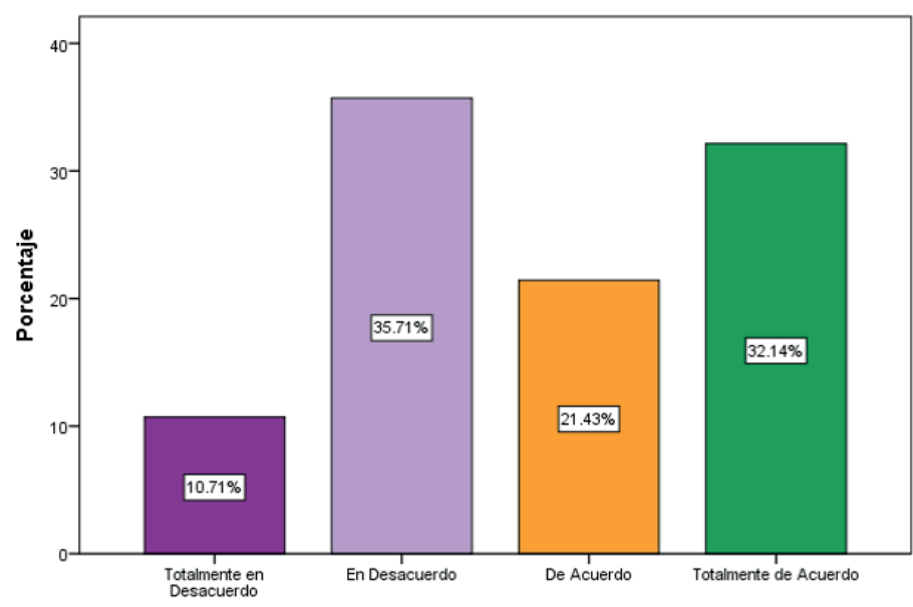

Figura 27. Laboratorios de computación con condiciones óptimas para desarrollar las prácticas asignadas con calidad

Fuente: propia a partir de encuesta aplicada a estudiantes 


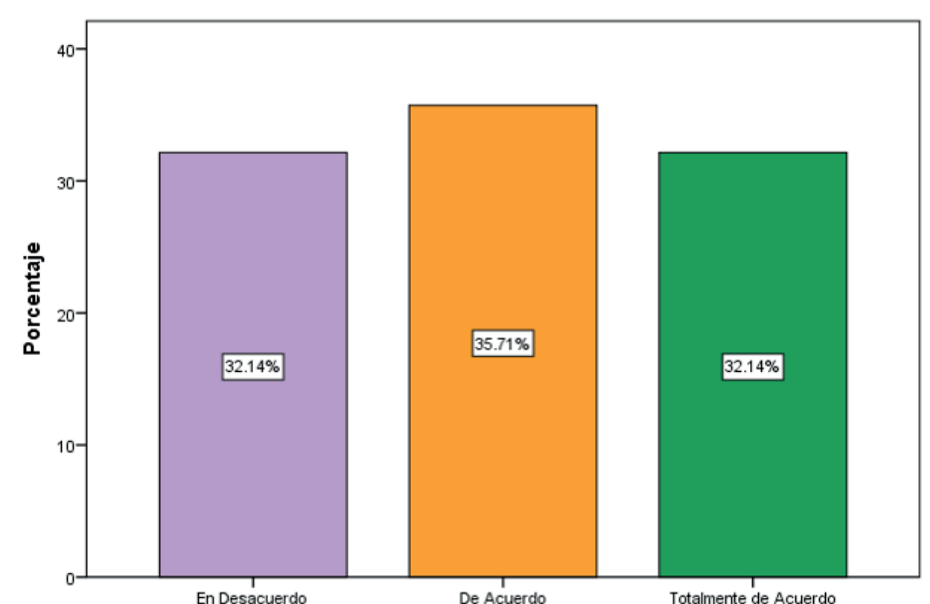

Figura 28. Los docentes usan una metodología adecuada para lograr un aprendizaje significativo

Fuente: propia a partir de encuesta aplicada a estudiantes

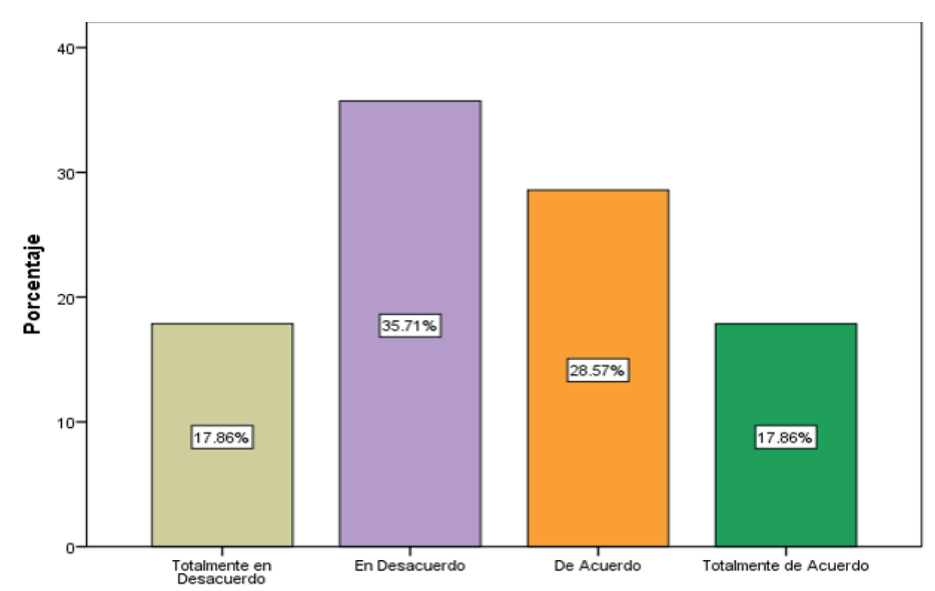

Figura 29. Sistema de becas universitario equitativo

Fuente: propia a partir de encuesta aplicada a estudiantes

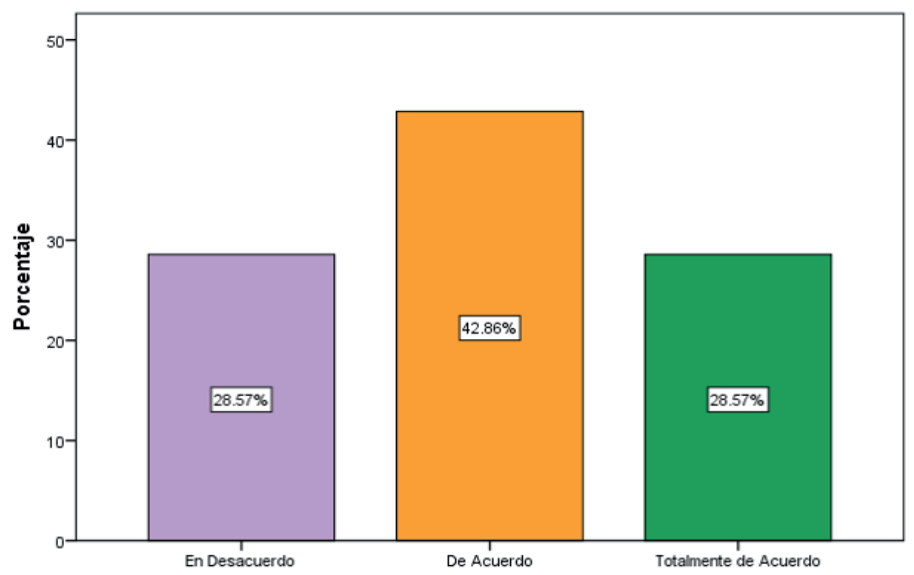

Figura 30. Biblioteca con excelente proceso de préstamos de material didáctico

Fuente: propia a partir de encuesta aplicada a estudiantes

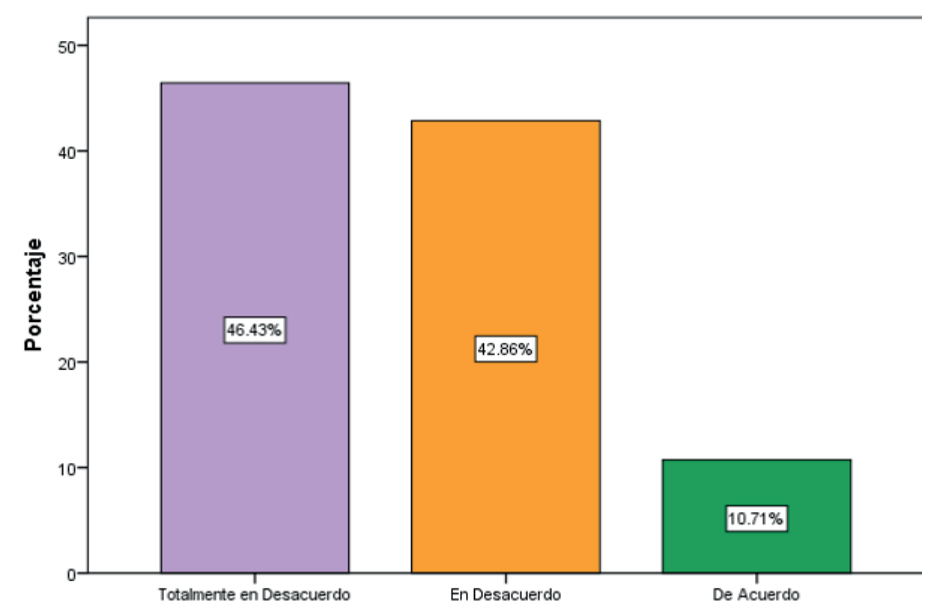

Figura 31. La universidad cuenta con un servicio adecuado de atención médica

Fuente: propia a partir de encuesta aplicada a estudiantes

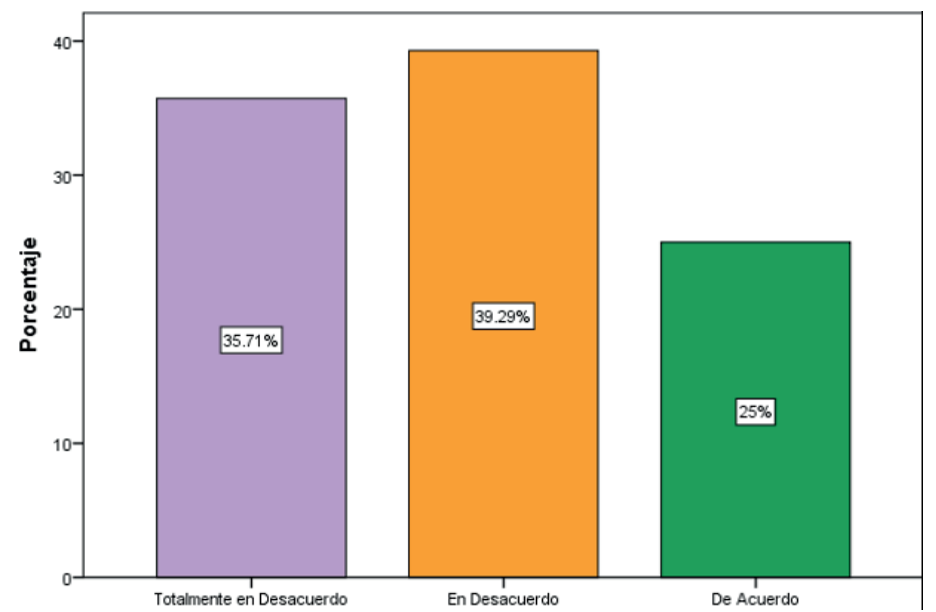

Figura 32. La universidad cuenta con un servicio adecuado de atención psicológica

Fuente: propia a partir de encuesta aplicada a estudiantes

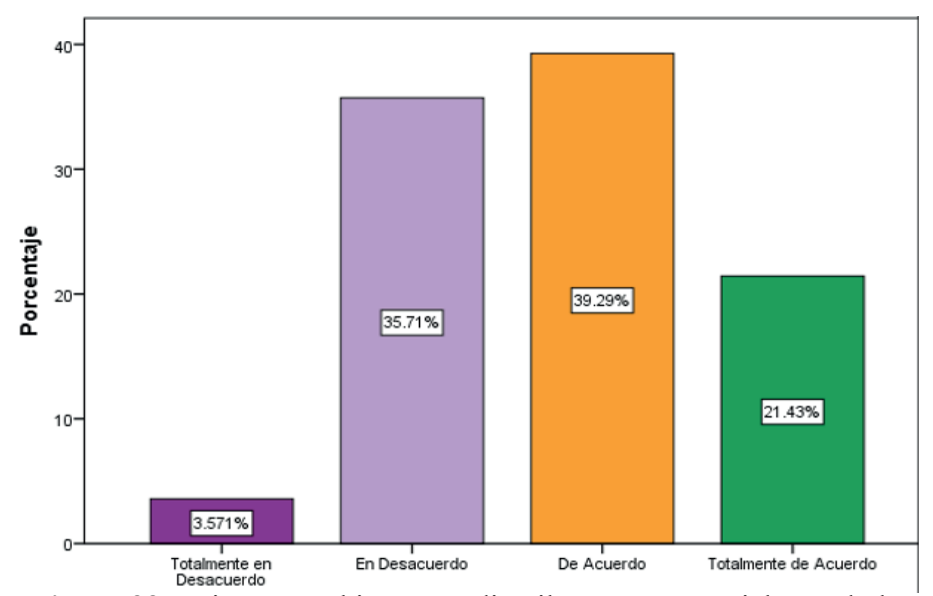

Figura 33. Existe un ambiente estudiantil con apoyo social cuando le aqueja un problema

Fuente: propia a partir de encuesta aplicada a estudiantes 


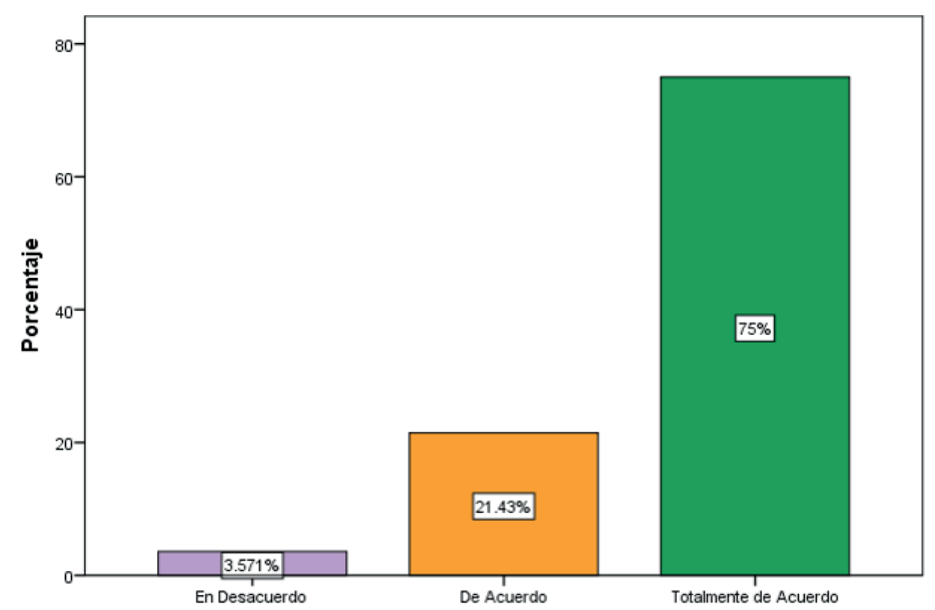

Figura 34. Es necesario aplicar a una prueba de admisión para ingresar a esta universidad

Fuente: propia a partir de encuesta aplicada a estudiantes

Los resultados anteriores muestran diversos factores que se deben mejorar por parte de la institución para ofrecer a los estudiantes mejores condiciones para que mediante la interrelación adecuada con los demás factores incidentes se logre mejorar el rendimiento académico de los estudiantes universitarios.

La mayoría de los Determinantes Institucionales se valoraron mediante un Análisis de Componentes Principales (ACP), donde el criterio de clasificación utilizado fue sexo y variables combinadas fueron los Determinantes Institucionales estudiados (Figura 35).

Se observó que más varones seleccionaron las variables DI6, DI9, DI11, DI12, DI18, DI19 Y DI20, que se refieren a factores institucionales relacionados con condiciones institucionales, ambiente estudiantil y relación docente-estudiante. Específicamente, opinan que el plan de estudio está planificado de acuerdo con las necesidades del mercado laboral, los métodos de evaluación normados por la universidad desarrollan un aprendizaje significativo, el horario en que se imparten las clases es adecuado, así como el tiempo asignado para desarrollar el contenido de las asignaturas del curso. También opinan que se cuenta con apoyo social cuando un estudiante tiene algún problema, hay excelentes relaciones afectivas y didácticas entre docentes y estudiantes.
Por otro lado, las mujeres seleccionaron factores institucionales como condiciones institucionales, servicios de apoyo, ambiente estudiantil y pruebas específicas de admisión a la universidad.

Opinan que han tenido dificultad para entender y estudiar algunas asignaturas, se cuentan con condiciones óptimas en las aulas, laboratorios y sala de medios, los docentes tienen una formación adecuada y usan una metodología calificada para lograr un aprendizaje significativo, los grupos de clase tienen la cantidad apropiada para desarrollar las clases, se cuenta con un sistema de becas equitativo y buen servicio en el proceso de préstamos de material didáctico en la biblioteca; existe un ambiente de solidaridad y compañerismo en la universidad y consideran necesario se aplique un examen de admisión para optar a la FAREM Matagalpa.

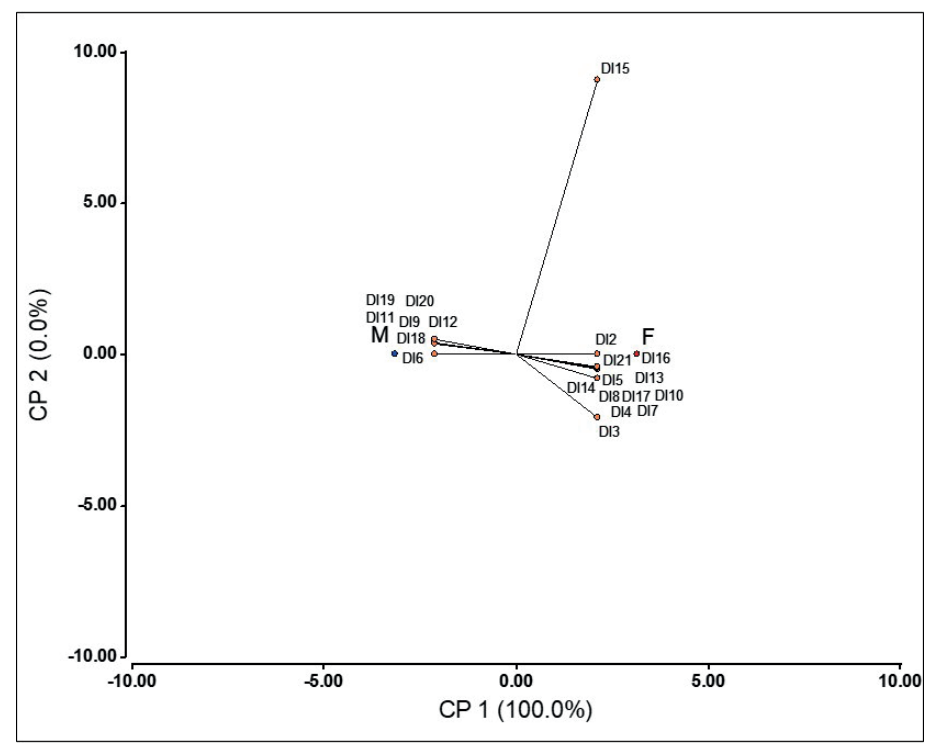

Figura 35. Otros Determinantes Institucionales clasificados por Sexo

Fuente: propia a partir de encuesta aplicada a estudiantes

Se han descrito los factores queinciden en el rendimiento académico de los estudiantes de Ing. en Sistemas de Información, clasificados por determinantes personales, sociales e institucionales; cabe recalcar que aunque fueron abordados de manera separada los tres se relacionan directa e indirectamente. Los resultados obtenidos son relavantes y pueden servir de base para 
profundizar sobre el tema, así se podría determinar la incidencia de los mismos en el rendimiento académico, esto daría las pautas necesarias para formular un plan de acción que mejore los resultados académicos de los estudiantes universitarios.

\section{CONCLUSIONES Y RECOMENDACIONES}

1. La mayoría de los estudiantes activos de la carrera de Ing. en Sistemas de Información se caracterizan por ser jóvenes, solteros, viven en el sector urbano, provienen de centros educativos públicos, no trabajan, viven con sus padres y prevalece el sexo femenino.

2. El rendimiento académico de los estudiantes activos presenta un promedio y un porcentaje de aprobados en limpio que sube a medida que el estudiante pasa de año. Casi la mitad de los estudiantes desertó de la carrera, la mayoría desertaron en el primer año de la carrera.

3. Los factores personales relevantes fueron competencia cognitiva, motivación, atribuciones causales, bienestar psicológico, asistencia a clases, inteligencia emocional, no existe relación entre promedio de secundaria y de universidad, y la última no se asocia con la nota de admisión.

4. Los factores sociales relevantes fueron entorno familiar, nivel educativo de padres, capital cultural y contexto socioeconómico.

5. Los factores institucionales determinantes fueron complejidad de estudios, condiciones institucionales, servicios de apoyo, relación estudiante-docente y pruebas de admisión.

6. Se recomienda tomar en cuenta los resultados de esta investigación para profundizar en el tema y tomar medidas para mejorar el rendimiento académico de los estudiantes universitarios.

7. Es recomendable realizar evaluaciones y observaciones continuas para determinar a tiempo las causas del mal rendimiento académico de algunos estudiantes, desde el inicio de la carrera.

\section{BIBLIOGRAFÍA}

Carrión, E. (2002). Validación de características al ingreso como predictores del rendimiento académico en la carrera de medicina. Revista Cubana de Educación Media Superior, 1(16).

Castejón, J., \& Pérez, A. (1998). Un modelo casual-explicativo sobre la influencia de las variables psicosociales en el rendimiento académico. Bordón. Revista de Pedagogía, 50(2), 171-185.

Cuasapaz, S., \& Rubio, M. A. (2013). Factores que influyen en el bajo rendimiento académico de los estudiantes de la escuela Sucre de la Ciudad de Tulcán en el periodo Marzo - Agosto 2013. Informe de Tesis, Tulcán.

Garbanzo, G. (2007). Factores asociados al rendimiento académico en estudiantes universitarios, una reflexión desde la calidad de la Educación Superior Pública. Revista Educación, 31(1), 43-63.

Gómez, D., Oviedo, R., \& Martínez, E. (2011). Factores que influyen en el rendimiento. Tecnociencia, $V(2), 90-97$.

Guido, J. D. (2016). Evaluación del Rendimiento Académico en la Carrera de Banca y Finanzas de la Facultad Multidisciplinaria de Carazo, en la UNAN Managua, en la Cohorte 2011 - 2015. Jinotepe, Nicaragua.

Hernández, R., Fernández, C., \& Baptista, P. (2010). Metodología de la Investigación. México: McGraw-Hill Interamericana.

Lozano, A. (2004). Factores personales, familiares y académicos que afectan al fracaso escolar en la Educación Secundaria. Revista Electrónica de Investigación Psicoeducativa y Psicopedagógica, 1(1), 43-66.

Meneses, W., Morillo, S., Navia, G., \& Grisales, M. (2013). Factores que afectan el rendimiento escolar en la institución educativa rural "Las Mercedes" desde la perspectiva de los actores institucionales. Plumilla Educativa, 433-452.

Miñano, P., Cantero, M., \& Castejón, J. (2008). Pre- 
dicción del Rendimiento Escolar de los Alumnos a partir de las aptitudes, el autoconcepto académico y las atribuciones causales. Horizontes Educacionales, 13(2), 11-23.

Montero, E., \& Villalobos, J. (2004). Factores institucionales, pedagógicos, psicosociales y sociodemográficos asociados al rendimientoacadémico y a la repetición estudiantil en la Universidad de Costa Rica. Costa Rica.

Ocaña, Y. (2011). Variables académicas que influyen en el rendimiento académico de los estudiantes universitarios. Investigación Educativa, 15(27), 165-179.

Pedroza (a), M. (2014). Metodología de la Investigación. La Organización y Diseño de la Investigación. Managua, Nicaragua.

Pedroza (b), M. (2015). Uso de Modelos ANOVA, ANACORR, ANARE. Managua, Nicaragua.

Pedroza, H., \& Dicovskyi, L. (2006). Sistema de análisis estadístico con SPSS. Managua, Nicaragua.

Pineda, E., de Alvarado, E., \& de Canales, F. (1994).
Metología de la Investigación. Washington, EUA: Organización Panamericana de la Salud.

Piura, J. (2006). Metodología de la Investigación Cientifica: Un Enfoque Integrador. Managua.

Tejedor, F., \& García, A. (2007). Causas del bajo rendimiento del estudiante universitario (en opinión de los profesores y alumnos). Propuestas de mejora en el marco del EEES. Revista de Educación, 443-473.

Torres, L., \& Rodríguez, N. (2006). Rendimiento académico y contexto familiar en estudiantes universitarios. Enseñanza e Investigación en Psicología, 11(002), 255-270.

Universidad Nacional Autónoma de Nicaragua. (2016). Rendimiento académico. Primer Semestre 2016. Managua, Nicaragua.

Vado, S., Mojica, P., \& Gómez, J. (2014). Indicadores de cobertura, eficiencia, eficacia, y eficiencia terminal para evaluar la gestión institucional desarrollada en la FAREM - Carazo para el período 2005 - 2010. Jinotepe, Nicaragua. 